\title{
A STABILIZED FINITE ELEMENT SCHEME FOR THE NAVIER-STOKES EQUATIONS ON QUADRILATERAL ANISOTROPIC MESHES
}

\begin{abstract}
MaLte BRAACK ${ }^{1}$
Abstract. It is well known that the classical local projection method as well as residual-based stabilization techniques, as for instance streamline upwind Petrov-Galerkin (SUPG), are optimal on isotropic meshes. Here we extend the local projection stabilization for the Navier-Stokes system to anisotropic quadrilateral meshes in two spatial dimensions. We describe the new method and prove an a priori error estimate. This method leads on anisotropic meshes to qualitatively better convergence behavior than other isotropic stabilization methods. The capability of the method is illustrated by means of two numerical test problems.
\end{abstract}

Mathematics Subject Classification. 35Q30, 65N30, 76D05.

Received April 18, 2007. Revised February 25, 2008 and March 31, 2008.

Published online August 12, 2008.

\section{INTRODUCTION}

The solution of partial differential equations on anisotropic meshes is of substantial importance for efficient solutions of problems with interior layers or boundary layers. For instance, in fluid dynamics at higher Reynolds number anisotropic meshes are usually used in order to resolve sharp gradients of velocity and pressure perpendicular to the boundary. Stabilized finite elements are well established in computational fluid dynamics, e.g. streamline upwind Petrov-Galerkin (SUPG), as introduced by Brooks and Hughes [13], or pressure stabilized Petrov-Galerkin (PSPG), see [23]. For the isotropic case, there is a wide range of methods and their analysis, as e.g. Tobiska and Lube [34], Hansbo and Szepessy [22], Franca and Frey [20], or the interior penalty method by Burman et al. [14]. As a first step towards (isotropic) projection-based stabilization the work of Codina [16] should be mentioned, where weighted global projections are added to the Galerkin terms.

But there is relatively few theoretical work done for anisotropic meshes. Formaggia and Perotto [18] derived a priori and a posteriori error estimates for elliptic problems on anisotropic triangular meshes where no stabilization is necessary. It is well-known that stabilized finite element schemes must be modified on anisotropic meshes. Usually, the anisotropic version differs from the isotropic version in the way to compute a characteristic mesh size parameter $h_{K}$ on each element $K$ which enters in the formulation as an important parameter. Taking this as the cell diameter is not the optimal choice as shown by Lube and Apel [28]. Micheletti et al. [32] propose for convection-diffusion problems and for the Stokes system to take instead the minimal eigenvalue $h_{K}:=\lambda_{K, \min }$ of the affine mapping of the reference triangle to the physical one, see [18]. The same is proposed by Codina

\footnotetext{
Keywords and phrases. Incompressible flow, Navier-Stokes equations, stabilized finite elements, anisotropic meshes.

1 Mathematisches Seminar, Christian-Albrechts-Universität zu Kiel, Ludewig-Meyn-Str. 4, 24098 Kiel, Germany.

braack@math.uni-kiel.de
} 
and Soto [17] in the case of the time-dependent Navier-Stokes equations with orthogonal subscale stabilization. They compare it successfully by a numerical comparison with other possibilities, e.g. to take $h$ as the maximum element length. Linss derived in [27] a particular choice of $h_{K}$ for a scalar convection diffusion problems on anisotropic meshes by a comparison with the residual free bubble approach. A numerical study shows a considerable reduction of the discretization error. Even less theoretical results are available for the Oseen system (as a standard linearization of the Navier-Stokes equations) on anisotropic meshes. The first error analysis is given by Lube et al. [30] in the case for SUPG/PSPG on hybrid meshes. Their modification in comparison to the isotropic version is also based on a different choice of $h_{K}$. On hybrid meshes with a presumed relation of the mesh sizes in the different coordinate directions based on the diffusion constant $\nu$, the parameter $h_{K}$ is chosen as $|K|^{1 / d}$.

In this work we propose a numerical scheme for fluid dynamics based on local projection stabilization (LPS) for anisotropic meshes. In particular, we give a proof for its optimality in the case of the Oseen system as a standard linearization of the Navier-Stokes system. The used techniques can probably be applied as well to an anisotropic version of subgrid stabilization of Guermond [21]. The isotropic version of local projection stabilization (LPS) was introduced in [3] in order to stabilize the saddle point structure of the Stokes system when equal-order finite elements are used. The method was extended in [4] for convective terms appearing for instance in the Navier-Stokes equation. An analysis is given in [8] for the Oseen system with equal-order (bi-)linear or (bi-)quadratic finite elements. Local projection stabilization techniques are already applied with large success to different fields of computational fluid dynamics, e.g., in 3D incompressible flows [10], compressible flows [33], reactive flows [11], parameter estimation [5,6] and optimal control problems [26]. Recently, an extension to arbitrary order and more general projections is derived by Matthies et al. [31].

The first step of formulating LPS on anisotropic quadrilateral meshes is published in [9] by considering the Stokes system. In the present work we address the by far more relevant and more difficult Oseen system where also the convective terms should be stabilized in such a way that the a priori error analysis remains optimal even when strongly stretched elements are used.

This work is structured as follows: in the following section we fix our notations of variables and spaces for the Oseen system and its Galerkin formulation. Well known asymptotic a priori estimates for the isotropic cases are presented and discussed in the context of anisotropic meshes. In Section 3, the LPS technique for isotropic meshes is shortly repeated and then extended to the anisotropic case on structured meshes and afterwards on unstructured quadrilateral meshes. The error analysis is presented Section 4. In Section 5, the proposed scheme is validated for the Navier-Stokes system on the basis of two model problems. In particular, we compare the anisotropic LPS variant with the classical isotropic version.

\section{Motivation of anisotropic a Priori estimates for the Oseen System}

\subsection{Oseen system}

The Oseen system in the domain $\Omega \subset \mathbb{R}^{d}$ for velocity $v$ and pressure $p$ consists of momentum and continuity equation:

$$
\begin{aligned}
\sigma v+(\beta \cdot \nabla) v-\mu \Delta v+\nabla p & =f \\
\operatorname{div} v & =0
\end{aligned}
$$

together with appropriate boundary conditions for $v$ (and eventually for $p$ ) on $\partial \Omega$. The absorption coefficient $\sigma$ is supposed to be non-negative, $\sigma \geq 0$. The convection field $\beta$ may vary in space but is solenoidal, $\operatorname{div} \beta=0$. The viscosity may also vary in space as long as $\mu(x)>0$ for each $x \in \Omega$. However, for ease of presentation we assume spatial constant $\mu$ and $\sigma$. We take in mind that the result in this work takes over to the case of varying coefficients with minor modifications. The right-hand side $f$ is supposed to be in the Hilbert space of square-integrable generalized functions $L_{2}(\Omega)$. 
For the analysis we restrict ourself to the case of homogeneous Dirichlet conditions for the velocities (no-slip conditions):

$$
\left.v\right|_{\partial \Omega}=0
$$

Due to the Dirichlet conditions for $v$ on the entire boundary, the pressure has to be normalized, for instance by the requirement of zero mean:

$$
\int_{\Omega} p(x) \mathrm{d} x=0
$$

In the numerical part of this work, we also consider mixed boundary conditions for $p$ and $v$.

\subsection{Galerkin formulation}

We embrace the two variables together in the variable $u:=\{v, p\}$. The natural function space is $X:=V \times Q$ with the Sobolev space of generalized functions with square-integrable derivatives and vanishing traces, $V:=$ $\left[H_{0}^{1}(\Omega)\right]^{d}$, and the Hilbert space $Q:=L_{2,0}(\Omega)$ consisting of $L_{2}(\Omega)$ functions with zero mean on $\Omega$. Test functions are denoted by Greek letters, for instance $\phi \in V$ as test function for the momentum equation and $\xi \in Q$ as test function for the continuity equation. Using the notation $(\cdot, \cdot)$ for the $L_{2}(\Omega)$-scalar product the corresponding Galerkin formulation reads

$$
u \in X: \quad a(u ; \phi, \xi)=(f, \phi) \quad \forall\{\phi, \xi\} \in X
$$

with the bilinear form

$$
a(u ; \phi, \xi):=(\sigma v, \phi)+((\beta \cdot \nabla) v, \phi)+(\mu \nabla v, \nabla \phi)-(p, \operatorname{div} \phi)+(\operatorname{div} v, \xi) .
$$

For the analysis of anisotropic effects we restrict to the two-dimensional case, $d=2$. The finite elements we consider in this work result from iso-parametric transformations $T_{K}: \widehat{K} \rightarrow K$ of bilinear functions $\widehat{\xi}$ on a reference cell $\widehat{K}$ :

$$
Q_{1, h}:=\left\{\xi \in C(\Omega, \mathbb{R}):\left.\xi\right|_{K}=\widehat{\xi} \circ T_{K}^{-1}\right\}
$$

The discrete pressure space $Q_{h}$ is the subspace of $Q_{1, h}$ with zero mean:

$$
Q_{h}:=\left\{\xi \in Q_{1, h}:(\xi, 1)=0\right\},
$$

and the discrete velocity space is the corresponding vector-valued space with vanishing traces:

$$
V_{h}:=\left\{\phi \in\left[Q_{1, h}\right]^{d}:\left.\phi\right|_{\partial \Omega}=0\right\} .
$$

The bilinear form $a(u ; \phi, \xi)$ is known to be unstable for such an equal-order interpolation of $V$ and $Q$ due to the violation of the discrete "inf-sup" condition [23], in particular for the finite element spaces under consideration in this work. A further instability is due to the dominant advective term. Stabilization is a standard tool to overcome this short-comings. In the next subsection, we state the well-known a priori results of stabilized equal-order finite elements for the Oseen system in order to clarify their deficits on anisotropic meshes. 


\subsection{Isotropic a priori results of stabilized schemes}

We denote the diameter of a cell $K$ by $h_{K}$. Then, the local quantity

$$
P e_{K}=|\beta|_{K, \infty} \frac{h_{K}}{\mu}
$$

is the so-called local Peclet number. The most interesting case is $P e_{K}>1$ (at least in certain cells $K$ ) which is also called the convection-dominant case. Residual-based methods, as for instance SUPG/PSPG, as well as projection-based schemes allow for equal-order finite elements of polynomial order $r$. For the estimates in this work, we use the notation " $a \lesssim b$ " for an upper bound $a \leq C b$ with a constant $C$ independent of the mesh size and aspect ratio. Moreover, this constant will not depend on the parameters $\sigma, \beta$ and $\mu$ of the problem, but may depend on the polynomial degree $r$. The expression $a \sim b$ is used if there holds $a \lesssim b$, and $b \lesssim a$ as well. It is well-known that the a priori estimate of SUPG/PSPG on shape-regular isotropic meshes is given by:

$$
\begin{aligned}
\left\|u-u_{h}\right\|_{r b s}^{2} & \lesssim \sum_{K \in \mathcal{T}_{h}} C_{K} h_{K}^{2 r}\|u\|_{H^{r+1}(K)}^{2}, \\
C_{K} & :=\mu+\|\beta\|_{L^{\infty}(K)} h_{K}+\sigma h_{K}^{2}
\end{aligned}
$$

for sufficiently smooth velocities and pressure $\left(H^{r+1}\right.$-regularity). Into the triplenorm $\|\cdot\|$ in $(2.2)$ the pressure enters via a $L_{2}$-term and in combination with the stream-wise derivative of the velocities

$$
\|u\|_{r b s}:=\left(\sigma\|v\|^{2}+\mu\|\nabla v\|^{2}+\gamma\|p\|^{2}+\sum_{K \in \mathcal{T}_{h}}\left(\delta_{K}\|(\beta \cdot \nabla) v+\nabla p\|_{K}^{2}+\gamma_{K}\|\operatorname{div} v\|_{K}^{2}\right)\right)^{1 / 2} .
$$

Here, $\gamma$ is a discrete "inf-sup" constant depending on the "inf-sup" constant of the continuous problem. Further cell-wise constant parameters as $\delta_{K}$ and $\gamma_{K}$ arise in the stabilized formulation. The estimate (2.2) is valid only for an "optimal choice" of these parameters, i.e. their asymptotic dependence on the local mesh size and the local Peclet number must be correct. We refer to [12] and Lube and Rapin [29] for the corresponding estimate for $P_{r} / P_{s}$ elements with $s<r$.

On quasi-uniform meshes with $h=\max \left\{h_{K}: K \in \mathcal{T}_{h}\right\} \sim h_{K}$ the a priori estimate (2.2) becomes for the interesting case of large Peclet number and moderate $\sigma$ (i.e. $M_{K} \sim\|\beta\|_{\infty} h$ ):

$$
\left\|u-u_{h}\right\|_{r b s} \lesssim\|\beta\|_{\infty}^{1 / 2} h^{r+1 / 2}\|u\|_{r+1}
$$

where $\|\cdot\|_{r+1}$ is the standard notation for the $H^{r+1}(\Omega)$-norm.

For local projection stabilization (LPS), the triple norm differs slightly, because certain fluctuations of the pressure gradient enter in the triple norm. This will be concretized in the following sections. However, we have for LPS on isotropic meshes qualitatively a very similar bound as (2.2) with a minor different definition of $M_{K}$, see [12]. The convergence on quasi-uniform meshes (2.3) is the same.

\subsection{A priori results on anisotropic meshes}

On anisotropic meshes, (2.2) and (2.3) are suboptimal, because $h_{K}$ is the cell diameter. Hence it is related to the mesh size with respect to the coordinate direction of maximal elongation. For instance in the case of a boundary layer with large second derivatives in $y$-direction, a Cartesian mesh should be much finer in $y$-direction, i.e. $h_{K, y} \ll h_{K, x}$, when $h_{K, x}$ and $h_{K, y}$ are the cell sizes in $x$ - and $y$-direction of a Cartesian mesh, respectively. More specifically, the optimal mesh sizes in the two coordinate directions should be

$$
\left(\frac{h_{K, x}}{h_{K, y}}\right)^{2} \approx \frac{\left\|\partial_{y y}^{2} v\right\|_{K}}{\left\|\partial_{x x}^{2} v\right\|_{K}}
$$


Now, in the estimate (2.3) the mesh size $h$ must be replaced by the maximal one, i.e. by $h_{x}=\max \left\{h_{K, x}: K \in\right.$ $\mathcal{T}_{h}$, so that (2.3) becomes for (bi-)linear elements:

$$
\left\|u-u_{h}\right\| \lesssim\|\beta\|_{\infty} h_{x}^{3 / 2}\left(\left\|\partial_{x} \nabla u\right\|+\left\|\partial_{y} \nabla u\right\|\right)
$$

if $u \in\left[H^{2}(\Omega)\right]^{d+1}$. Here, $\|\cdot\|$ stands for $\|\cdot\|_{r b s}$ as well as for $\|\cdot\|_{\text {lps }}$ (defined later). Obviously, the second derivatives of $u$ in the estimate $(2.5)$ are not well balanced. In particular, the term $h_{x}^{3 / 2}\left\|\partial_{y} \nabla u\right\|$ would be the dominant one when the second derivative in $y$-direction is much smaller than the one in $x$-direction and the mesh sizes are designed in the optimal way (2.4).

Formaggia and Perotto derived in [18] an a priori estimate for elliptic problems on anisotropic meshes. For ease of presentation, we simplify their result for a moment to the Poisson problem $-\Delta v=f$ on a Cartesian (triangular) mesh. Their results for linear elements reads as

$$
\left\|\nabla\left(v-v_{h}\right)\right\| \lesssim \sum_{K \in \mathcal{T}_{h}}\left(\frac{h_{K, x}^{4}}{h_{K, y}^{2}}\left\|\partial_{x x}^{2} v\right\|_{K}^{2}+2 h_{K, x}^{2}\left\|\partial_{x y}^{2} v\right\|_{K}^{2}+h_{K, y}^{2}\left\|\partial_{y y}^{2} v\right\|_{K}^{2}\right)^{1 / 2}
$$

We observe here, that $h_{K, x}$ and $h_{K, y}$ are multiplied by the corresponding spatial derivatives. The contributions are well equilibrated under the condition (2.4), at least in the usual situation that the mixed second derivative is much smaller than the maximal pure one.

Formaggia et al. [19] analyzed the SUPG stabilization for advection-diffusion-reaction problems on triangular meshes. It results that the stabilization constant should be computed with respect to the smallest elongation of the mesh cells. This gives an a priori estimate with an appropriate scaling of the different spatial derivatives for quite arbitrary anisotropic meshes. For ease of presentation, let us shortly restrict their results on Cartesian meshes with $h_{y} \leq h_{x}$. Then their results for SUPG stabilized advection-diffusion-reaction problems reads as:

$$
\left\|u-u_{h}\right\|_{r b s}^{2} \lesssim \sum_{K \in \mathcal{T}_{h}} C_{K}\left(\frac{h_{K, x}^{4}}{h_{K, y}}\left\|\partial_{x x}^{2} v\right\|_{K}^{2}+2 h_{K, y} h_{K, x}^{2}\left\|\partial_{x y}^{2} v\right\|_{K}^{2}+h_{K, y}^{3}\left\|\partial_{y y}^{2} v\right\|_{K}^{2}\right)
$$

Here, the cell-wise parameter $C_{K}$ is given by $C_{K}=\mu h_{K, y}^{-1} \max \left(1, P e_{K}\right)$ with the local Peclet number with respect to the small mesh size, $P e_{K}:=\|\beta\|_{K, \infty} h_{K, y} \mu^{-1}$.

Lube et al. give in [30] an anisotropic error analysis for SUPG/PSPG for the Oseen system on hybrid meshes with a presumed relation of the mesh sizes in the different coordinate directions based on the diffusion constant $\mu$.

In this work, we will formulate an anisotropic modification of LPS so that the following estimate holds:

$$
\begin{aligned}
\left\|u-u_{h}\right\|_{\text {lps }}^{2} \lesssim & \sum_{K \in \mathcal{T}_{h}}\left\{C_{K, p}\left(h_{K, y}^{-1} h_{K, x}^{4}\left\|\partial_{x} \nabla p\right\|_{K}^{2}+h_{K, y}^{3}\left\|\partial_{y} \nabla p\right\|_{K}^{2}\right)\right. \\
& \left.+C_{K, v}\left(h_{K, y}^{-1} h_{K, x}^{4}\left\|\partial_{x} \nabla v\right\|_{K}^{2}+h_{K, y}^{3}\left\|\partial_{y} \nabla v\right\|_{K}^{2}\right)\right\}
\end{aligned}
$$

with quantities $C_{K, p}, C_{K, v}$ specified later and depending mainly on the local Peclet number. If the mesh sizes $h_{x}$ and $h_{y}$ are well balanced according to (2.4), both contributions from $\left\|\partial_{x} \nabla u\right\|_{K}$ and $\left\|\partial_{y} \nabla u\right\|_{K}$ in (2.8) are well equilibrated and the bound is much better that (2.5). Comparing our result (2.8) with the isotropic version (2.5) we see that the estimate is better by a factor of $\left(h_{K, x} / h_{K, y}\right)^{3 / 2}$. For large aspect ratios, this is an enormous gain. A comparison with (2.7) (for a scalar advection-diffusion-reaction equation) shows that we obtain a very similar equilibration of the contributions with respect to the spatial derivatives even for the Oseen system.

After a short presentation of LPS on isotropic meshes, we will generalize this technique to the case of anisotropic meshes on quadrilateral meshes. A stability proof and an a priori estimate will be given for Cartesian and rotated meshes. 

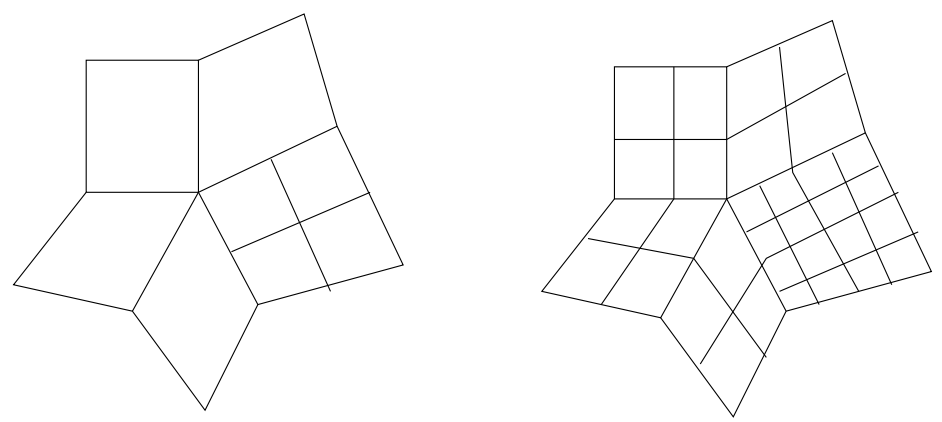

Figure 1. Possible meshes consisting of patches, $\mathcal{T}_{2 h}$ (left) and $\mathcal{T}_{h}$ (right).

\section{LOCAL PROJECTION STABILIZATION}

The idea of LPS, see [4], consists of adding inconsistent stabilization terms $s_{h}\left(u_{h} ; \phi, \xi\right)$ to the Galerkin form so that the stabilized discrete system is of the following form: Find $u_{h}=\left\{v_{h}, p_{h}\right\} \in X_{h}:=V_{h} \times Q_{h}$ such that

$$
a\left(u_{h} ; \phi, \xi\right)+s_{h}\left(u_{h} ; \phi, \xi\right)=(f, \phi) \quad \forall\{\phi, \xi\} \in X_{h} .
$$

The term $s_{h}\left(u_{h} ; \phi, \xi\right)$ is different for isotropic meshes and anisotropic meshes. However, in both cases it is based on a local projection described in the following.

The mesh $\mathcal{T}_{h}$ is supposed to be constructed by patches of quadrilaterals. A coarser mesh $\mathcal{T}_{2 h}$ is obtained by one global coarsening of $\mathcal{T}_{h}$. The correspondence between these two meshes is as follows: each quadrilateral $P \in \mathcal{T}_{2 h}$ is cut into four new quadrilaterals (dividing all lengths of edges of $P$ by 2 ) in order to obtain the fine partition $\mathcal{T}_{h}$. Due to this construction we can associate to each cell $K \in \mathcal{T}_{h}$ a corresponding patch $P=P(K) \in \mathcal{T}_{2 h}$ with $K \subset P$. In Figure 1 we show such a mesh schematically. Such meshes are also the basis of the two-level scheme of John et al. [24,25]. For the formulation of this projection method, hanging nodes are allowed. The discrete solution is interpolated on these irregular nodes by the values on the regular nodes of the corresponding edge so that the solution remains continuous. In other words, no degrees of freedom are associated to these irregular nodes. This standard procedure is described e.g. by Ciarlet [15] and is applicable to arbitrary order $r \geq 1$. However, in the analysis of this work, we restrict to the case of anisotropic Cartesian meshes without such irregular nodes.

The space $Q_{2 h}^{\text {disc }}$ consists of patch-wise constants, but discontinuous across patches $P \in \mathcal{T}_{2 h}$ :

$$
Q_{2 h}^{\text {disc }}:=\left\{\xi \in L^{2}(\Omega):\left.\xi\right|_{P} \equiv \text { const. } \forall P \in \mathcal{T}_{2 h}\right\}
$$

The projection

$$
\pi_{h} \quad: \quad L^{2}(\Omega) \rightarrow Q_{2 h}^{\text {disc }}
$$

is defined as the patch-wise mean. On a patch $P \in \mathcal{T}_{2 h}$ we have:

$$
\left.\pi_{h} q\right|_{P}:=\frac{1}{|P|} \int_{P} q(x) \mathrm{d} x .
$$

In particular, it satisfies the following orthogonality property:

$$
\left(q-\pi_{h} q, \xi\right)=0 \quad \forall \xi \in Q_{2 h}^{\mathrm{disc}} .
$$


The difference between the identity $I$ and $\pi_{h}$,

$$
\kappa_{h}:=I-\pi_{h},
$$

is a fluctuation filter entering in the stabilization. Its concrete form is specified in the following subsections.

\subsection{The case of isotropic meshes}

On isotropic meshes the stabilization term $s_{h}(\cdot ; \cdot, \cdot)$ in $(3.1)$ is of the form:

$$
s_{h}^{i s o}\left(u_{h} ; \phi, \xi\right):=\left(\kappa_{h} \nabla p_{h}, \alpha \nabla \xi\right)+\left(\kappa_{h}\left(\nabla v_{h}\right), \delta \nabla \phi\right)
$$

The quantities $\alpha$ and $\delta$ are patch-wise constants, depending on the local Peclet number. If the flow is locally transport dominated, i.e. $P e_{K}>1$ on cell $K$, the stabilization parameters are chosen on the associated patch $P=P(K)$ as $\left.\left.\alpha\right|_{P} \sim \delta\right|_{P} \sim h_{P} \sim h_{K}$.

Due to the orthogonality property (3.2) it holds

$$
\left(\kappa_{h} \nabla p, \alpha \nabla \xi\right)=\left(\kappa_{h} \nabla p, \alpha \kappa_{h} \nabla \xi\right) \quad \forall p, \xi \in Q
$$

The same type of orthogonality holds for the stabilization for the velocities. As a consequence, the stabilization term (3.3) is symmetric.

Instead of the full gradient $\nabla v$ in the definition (3.3) of the stabilizing term, the stream-wise derivative together with additional control over the fluctuations of the divergence of $v$ can be chosen:

$$
{\widetilde{s_{h}}}^{i s o}\left(u_{h} ; \phi, \xi\right):=\left(\kappa_{h} \nabla p_{h}, \alpha \nabla \xi\right)+\left(\kappa_{h}\left(\beta \cdot \nabla v_{h}\right), \delta \beta \cdot \nabla \phi\right)+\left(\kappa_{h}\left(\operatorname{div} v_{h}\right), \gamma \operatorname{div} \phi\right)
$$

We refer to [8] for more details. For comparison, we recall a standard residual-based stabilization consisting of PSPG and SUPG, see [20,22,34]:

$$
s_{h}^{r b s}\left(u_{h} ; \phi, \xi\right):=\sum_{K \in \mathcal{T}_{h}}\left\{\left(\operatorname{div} v_{h}, \gamma \operatorname{div} \phi\right)_{K}+\left(\sigma v_{h}+(\beta \cdot \nabla) v_{h}-\mu \Delta v_{h}+\nabla p_{h}-f, \alpha \nabla \xi+\delta(\beta \cdot \nabla) \phi\right)_{K}\right\}
$$

with (different) cell-wise constant parameters $\alpha, \delta, \gamma$. Control over the divergence div $v_{h}$ is included in residualbased stabilization as for LPS when only stream-wise derivatives of $v_{h}$ are stabilized.

\subsection{Local projection stabilization on anisotropic Cartesian meshes}

The local projection stabilization for Oseen for anisotropic meshes aligned with the coordinate axes becomes:

$$
s_{h}(u ; \phi, \xi):=\left(M_{p} \kappa_{h}\left(\nabla p_{h}\right), M_{p} \nabla \xi\right)+\left(M_{v} \kappa_{h}(\nabla v), M_{v} \nabla \phi\right)
$$

with the diagonal matrices $M_{p}, M_{v}$ are given by

$$
M_{p}=\operatorname{diag}\left(\alpha_{x}^{1 / 2}, \alpha_{y}^{1 / 2}\right), \quad M_{v}=\operatorname{diag}\left(\delta_{x}^{1 / 2}, \delta_{y}^{1 / 2}\right)
$$

and patch-wise constant parameters $\alpha_{x}, \alpha_{y}, \delta_{x}, \delta_{y}$. Because $M_{p}$ and $M_{v}$ are diagonal matrices, the stabilization can also be written as:

$$
s_{h}(u ; \phi, \xi):=\left(\kappa_{h}\left(\partial_{x} p_{h}\right), \alpha_{x} \partial_{x} \xi\right)+\left(\kappa_{h}\left(\partial_{y} p_{h}\right), \alpha_{y} \partial_{y} \xi\right)+\left(\kappa_{h}\left(\partial_{x} v_{h}\right), \delta_{x} \partial_{x} \phi\right)+\left(\kappa_{h}\left(\partial_{y} v_{h}\right), \delta_{y} \partial_{y} \phi\right) .
$$


In order to derive the announced estimate (2.8) we have to determine the scaling of the stabilization parameters appropriately. It turns out that the optimal choice of the stabilization parameters depends on the minimal Peclet number:

$$
\mathrm{Pe}_{\min , K}:=\frac{\min \left(h_{K, y}, h_{K, x}\right)\|\beta\|_{\infty, K}}{\mu} .
$$

For the isotropic case, this characteristic number is the usual local Peclet number. Since the mesh size may vary from cell to cell, this is a local quantity.

The analysis in the next section will show that the following choice of the parameters $\alpha_{K, x}:=\left.\alpha_{x}\right|_{K}, \alpha_{K, y}:=$ $\alpha_{K, y}, \delta_{K, x}:=\left.\delta_{x}\right|_{K}$ and $\delta_{K, y}:=\left.\delta_{y}\right|_{K}$ are optimal:

$$
\begin{aligned}
\alpha_{K, 0} & :=\mu^{-1} \min \left(1, \mathrm{Pe}_{\min , K}^{-1}\right), \\
\alpha_{K, x} & =h_{K, x}^{2} \alpha_{K, 0}, \\
\alpha_{K, y} & =h_{K, y}^{2} \alpha_{K, 0}, \\
\delta_{K, x} & =\|\beta\|_{\infty, K}^{2} \alpha_{K, x}, \\
\delta_{K, y} & =\|\beta\|_{\infty, K}^{2} \alpha_{K, y} .
\end{aligned}
$$

The convection dominant case $\mathrm{Pe}_{\mathrm{min}, K}^{-1} \ll 1$ for certain cells $K$ is of course the most interesting one.

For residual type stabilization schemes (SUPG, PSPG), the difference between the isotropic and the anisotropic case consists in a different choice of the stabilization parameters, see e.g. Formaggia et al. [19], while the additional terms remain isotropic. The stabilization parameter in [19] is identical to $\alpha_{K, y}$. However, for LPS, the proposed stabilization itself is anisotropic. The parameters $\delta_{K, x}$ and $\delta_{K, y}$ must differ from $\alpha_{K, x}$ and $\alpha_{K, y}$, respectively, by the factor $\|\beta\|_{\infty, K}^{2}$ in order to obtain optimality.

\subsection{Extension to meshes under affine linear maps}

Now we allow the patches $K \in \mathcal{T}_{2 h}$ to be mapped by a standard invertible affine linear map from a reference quadrilateral $\widehat{K}$ to the patch $K, T_{K}: \widehat{x} \mapsto x$. Following the notations in [18] such a map can be formulated as

$$
T_{K} \widehat{x}=t_{K}+M_{K} \widehat{x}
$$

with a shift vector $t_{K} \in \mathbb{R}^{2}$ and a matrix of the form $M_{K}=B_{K} Z_{K} \in \mathbb{R}^{2 \times 2}$. The matrix $Z_{K}$ is an orthonormal matrix and $B_{K}$ is symmetric positive definite with eigenvalues $\lambda_{K, 1} \geq \lambda_{K, 2}>0$ and eigenvectors $r_{1}, r_{2}$, sampled together in the matrices $\Lambda_{K}=\operatorname{diag}\left(\lambda_{K, 1}, \lambda_{K, 2}\right)$ and $R_{K}=\left[r_{1}, r_{2}\right]$. Hence

$$
B_{K}=R_{K}^{T} \Lambda_{K} R_{K}
$$

The stabilization term is still of the form (3.4) but with a different definition of the matrices $M_{p}$ and $M_{v}$. These become on a patch $K \in \mathcal{T}_{h}$ :

$$
\begin{aligned}
\left.M_{p}\right|_{K} & =\alpha_{K, 0}^{1 / 2} M_{K}, \\
\left.M_{v}\right|_{K} & =\left.\|\beta\|_{\infty, K} M_{p}\right|_{K} .
\end{aligned}
$$

In the case of a Cartesian mesh, it holds $Z_{K}=R_{K}=I$ and $\Lambda_{K}=\operatorname{diag}\left(h_{x}, h_{y}\right)$. It is easy to check, that we recover the previous case of Cartesian grids with the same orientation as the coordinate axes, i.e. $M_{p}$ and $M_{v}$ are given by (3.5). 


\section{ERROR ANALYSIS}

\subsection{Stability}

The a priori estimate (2.2) was shown in [8] for piecewise bilinear $(r=1)$ and quadratic $(r=2)$ elements for the triple norm:

$$
\|u\|_{\mathrm{lps}}:=\left(\sigma\|v\|^{2}+\mu\|\nabla v\|^{2}+s_{h}(u ; u)\right)^{1 / 2} .
$$

In the following analysis of the anisotropic version, we focus on the case $r=1$ so that the presentation remains simpler. However, the analysis holds for arbitrary order (but sub-optimal for $r \geq 2$ ). At first, we formulate the stability property and the "perturbed Galerkin orthogonality" property of the discrete operator $a(\cdot ; \cdot)+s_{h}(\cdot ; \cdot)$ :

Theorem 4.1. For all $u \in X$ it holds the stability property

$$
a(u ; u)+s_{h}(u ; u)=\|u\|_{\mathrm{lps}} .
$$

If $u \in X$ is the solution of (2.1) and $u_{h} \in X_{h}$ the solution of the discrete problem (3.1), then it holds:

$$
a\left(u-u_{h} ; \varphi\right)=s_{h}\left(u_{h} ; \varphi\right) \quad \forall \varphi \in X_{h}
$$

Proof. The stability follows immediate after integration by parts keeping in mind that $\beta$ is solenoidal. The perturbed orthogonality property is obtained by subtracting (3.1) from (2.1). Both properties are independent of the specific structure of $s_{h}(\cdot ; \cdot)$ as long it is symmetric.

\section{2. $\boldsymbol{L}_{\mathbf{2}}$-orthogonal interpolation and fluctuations on anisotropic Cartesian meshes}

In this part, we develop an $L_{2}$-orthogonal interpolation on anisotropic Cartesian meshes. This will be needed to analyze LPS for meshes as treated in Section 3.2. The mesh is assumed to be finer in $y$-direction:

$$
h_{K, y}<h_{K, x}
$$

We only need the important interior angle condition for neighbor cells $K, L \in \mathcal{T}_{h}$ :

$$
h_{K, x} \sim h_{L, x} \quad \text { and } \quad h_{K, y} \sim h_{L, y}
$$

This condition implies that the mesh sizes with respect to the $x$-direction of neighbor cells are of the same order. The same should hold for the $y$-directions. Although we allow for varying cells sizes in $x$-direction (and in $y$-direction), this change must be moderate from one cell to the next one.

A key ingredient for showing that the (inconsistent) stabilization term has an appropriate asymptotic behavior on anisotropic meshes is the existence of an anisotropic $L_{2}$-orthogonal interpolation operator $\mathcal{J}_{h}$ :

Lemma 4.2. It exists an interpolation operator $\mathcal{J}_{h}: V \rightarrow V_{h}$ with the orthogonality property

$$
\left(v-\mathcal{J}_{h} v, \psi\right)=0 \quad \forall v \in V, \forall \psi \in Q_{2 h}^{\text {disc }},
$$

which satisfies on each cell $K \in \mathcal{T}_{h}$ and its associated patch $P=P(K) \in \mathcal{T}_{2 h}$ the following stability properties

$$
\begin{aligned}
\left\|\partial_{x} \mathcal{J}_{h} u\right\|_{K} & \lesssim\left\|\partial_{x} u\right\|_{P}+\frac{h_{K, y}}{h_{K, x}}\left\|\partial_{y} u\right\|_{P} \\
\left\|\partial_{y} \mathcal{J}_{h} u\right\|_{K} & \lesssim h_{K, y}^{-1} h_{K, x}\left\|\partial_{x} u\right\|_{P}+\left\|\partial_{y} u\right\|_{P}
\end{aligned}
$$


as well as the following approximation properties for $u \in H^{2}(\Omega)$

$$
\begin{aligned}
\left\|u-\mathcal{J}_{h} u\right\|_{K} & \lesssim h_{K, x}^{2}\left\|\partial_{x} \nabla u\right\|_{P}+h_{K, y}^{2}\left\|\partial_{y} \nabla u\right\|_{P} \\
\left\|\partial_{x}\left(u-\mathcal{J}_{h} u\right)\right\|_{K} & \lesssim h_{K, x}\left\|\partial_{x} \nabla u\right\|_{P}+h_{K, x}^{-1} h_{K, y}^{2}\left\|\partial_{y} \nabla u\right\|_{P}, \\
\left\|\partial_{y}\left(u-\mathcal{J}_{h} u\right)\right\|_{K} & \lesssim h_{K, y}^{-1} h_{K, x}^{2}\left\|\partial_{x} \nabla u\right\|_{P}+h_{K, y}\left\|\partial_{y} \nabla u\right\|_{P} .
\end{aligned}
$$

Proof. We follow the idea in [8] and construct

$$
\mathcal{J}_{h}:=\mathcal{B}_{h}+\mathcal{M}_{h}
$$

as a sum of an interpolation operator $\mathcal{B}_{h}$ which is the anisotropic version of the Scott-Zhang operator $($ see $[1,2,7])$ fulfilling certain anisotropic interpolation properties specified in a short while, and a corrector $\mathcal{M}_{h}$ which insures the orthogonality property (4.2). In [8] (isotropic version) the operator $\mathcal{M}_{h}$ is build in such a way that for $u \in V$ and $\psi \in Q_{2 h}^{\text {disc }}$ it holds:

$$
\left(\mathcal{M}_{h} u, \psi\right)=\left(u-\mathcal{S} Z_{h} u, \psi\right)
$$

and $\left\|\mathcal{M}_{h} u\right\|_{K} \lesssim\left\|u-\mathcal{S} Z_{h} u\right\|_{P}$, where $\mathcal{S} Z_{h}$ is the isotropic Scott-Zhang interpolator. However, since no specific feature of $\mathcal{S} Z_{h}$ is used in [8], the construction take over to the case where we replace $\mathcal{S} Z_{h}$ in (4.8) by the anisotropic version $\mathcal{B}_{h}$. Hence, we obtain the orthogonality property (4.2) and

$$
\left\|\mathcal{M}_{h} u\right\|_{K} \lesssim\left\|u-\mathcal{B}_{h} u\right\|_{P}
$$

For details on the construction of $\mathcal{M}_{h}$ we refer to [8]. The operator $\mathcal{B}_{h}$ satisfies (see [7]):

$$
\begin{aligned}
\left\|\partial_{x} \mathcal{B}_{h} u\right\|_{K} & \lesssim\left\|\partial_{x} u\right\|_{P}+\frac{h_{K, y}}{h_{K, x}}\left\|\partial_{y} u\right\|_{P}, \\
\left\|\partial_{y} \mathcal{B}_{h} u\right\|_{K} & \lesssim\left\|\partial_{y} u\right\|_{P}, \\
\left\|u-\mathcal{B}_{h} u\right\|_{K} & \lesssim h_{K, x}\left\|\partial_{x} u\right\|_{P}+h_{K, y}\left\|\partial_{y} u\right\|_{P},
\end{aligned}
$$

as well as the following approximation properties for $u \in H^{2}(\Omega)$

$$
\begin{aligned}
\left\|u-\mathcal{B}_{h} u\right\|_{K} & \lesssim h_{K, x}^{2}\left\|\partial_{x} \nabla u\right\|_{P}+h_{K, y}^{2}\left\|\partial_{y} \nabla u\right\|_{P}, \\
\left\|\partial_{x}\left(u-\mathcal{B}_{h} u\right)\right\|_{K} & \lesssim h_{K, x}\left\|\partial_{x} \nabla u\right\|_{P}+h_{K, x}^{-1} h_{K, y}^{2}\left\|\partial_{y} \nabla u\right\|_{P}, \\
\left\|\partial_{y}\left(u-\mathcal{B}_{h} u\right)\right\|_{K} & \lesssim h_{K, x}\left\|\partial_{x} \nabla u\right\|_{P}+h_{K, y}\left\|\partial_{y} \nabla u\right\|_{P} .
\end{aligned}
$$

Now, we turn to the stability and approximation properties of the sum $\mathcal{J}_{h}=\mathcal{B}_{h}+\mathcal{M}_{h}$. The $L_{2}$-estimate $(4.5)$ is a direct consequence of (4.9) and (4.13):

$$
\left\|u-\mathcal{J}_{h} u\right\|_{K} \leq\left\|u-\mathcal{B}_{h} u\right\|_{K}+\left\|\mathcal{M}_{h} u\right\|_{K} \lesssim\left\|u-\mathcal{B}_{h} u\right\|_{K} .
$$

Stability (4.3) and (4.4) is obtained due to stability of $\mathcal{B}_{h}\left((4.10)\right.$ and (4.11)) and due to the estimate of $\left\|\partial_{x} \mathcal{M}_{h} u\right\|$ and $\left\|\partial_{y} \mathcal{M}_{h} u\right\|$ by an inverse inequality:

$$
\begin{aligned}
\left\|\partial_{x} \mathcal{M}_{h} u\right\|_{K} & \lesssim h_{K, x}^{-1}\left\|\mathcal{M}_{h} u\right\|_{K} \\
& \lesssim h_{K, x}^{-1}\left\|u-\mathcal{B}_{h} u\right\|_{K} \\
& \lesssim\left\|\partial_{x} u\right\|_{P(K)}+h_{K, x}^{-1} h_{K, y}\left\|\partial_{y} u\right\|_{P(K)}
\end{aligned}
$$


The bound (4.4) is obtained analogously. The estimate (4.6) is obtained by the inverse estimate (4.16) and (4.13):

$$
\left\|\partial_{x} \mathcal{M}_{h} u\right\|_{K} \lesssim h_{K, x}\left\|\partial_{x} \nabla u\right\|_{P(K)}+h_{K, x}^{-1} h_{K, y}^{2}\left\|\partial_{y} \nabla u\right\|_{P(K)}
$$

The proof of (4.7) is similar but based on the inverse estimate

$$
\left\|\partial_{y} \mathcal{M}_{h} u\right\|_{K} \lesssim h_{K, y}^{-1} h_{K, x}^{2}\left\|\partial_{x} \nabla u\right\|_{P(K)}+h_{K, y}\left\|\partial_{y} \nabla u\right\|_{P(K)}
$$

Remark 4.3. These estimates are qualitatively very similar to the anisotropic interpolation result in [18]. In particular, we obtain the same factor $h_{K, y}^{-1} h_{K, x}$ in front of the pure $x$-derivative in (4.7) reflecting the degree of anisotropy and seems to be suboptimal, because the anisotropic interpolant $\mathcal{B}_{h}$ does not need this factor and is only due to the additional orthogonality property (4.2). However, we will see that this factor comes into play for the local projection stabilization at another place independently of the approximation properties of $\mathcal{J}_{h}$.

Lemma 4.4. We consider the interpolant $\mathcal{J}_{h} u$ of Lemma 4.2 and the triple norm $\|\cdot\|_{\text {lps }}$ with the stabilization term defined in (3.4) and the following relation for the parameters

$$
\alpha_{K, x}=\alpha_{K, y} h_{K, x}^{2} h_{K, y}^{-2} \quad \text { and } \quad \delta_{K, x}=\delta_{K, y} h_{K, x}^{2} h_{K, y}^{-2}
$$

Then we obtain the following upper bound:

$$
\begin{aligned}
\left\|u-\mathcal{J}_{h} u\right\|_{\mathrm{lps}}^{2} \lesssim & \sum_{K \in \mathcal{T}_{h}}\left\{\alpha_{K, x} h_{K, x}^{2}\left\|\partial_{x} \nabla p\right\|_{K}^{2}+\alpha_{K, y} h_{K, y}^{2}\left\|\partial_{y} \nabla p\right\|_{K}^{2}\right. \\
& \left.+\left(\sigma+\left(\mu+\delta_{K, y}\right) h_{K, y}^{-2}\right) \cdot\left(h_{K, x}^{4}\left\|\partial_{x} \nabla v\right\|_{K}^{2}+h_{K, y}^{4}\left\|\partial_{y} \nabla v\right\|_{K}^{2}\right)\right\} .
\end{aligned}
$$

Proof. By the use of the preceding lemma we obtain with $h_{K, y} \leq h_{K, x}$ :

$$
\sigma\left\|v-\mathcal{J}_{h} v\right\|^{2}+\mu\left\|\nabla\left(v-\mathcal{J}_{h} v\right)\right\|^{2} \lesssim \sum_{K \in \mathcal{T}_{h}}\left\{\left(\sigma h_{K, x}^{2}+\mu h_{K, x}^{2} h_{K, y}^{-2}\right) h_{K, x}^{2}\left\|\partial_{x} \nabla v\right\|^{2}+\left(\sigma h_{K, y}^{2}+\mu\right) h_{K, y}^{2}\left\|\partial_{y} \nabla v\right\|^{2}\right\} .
$$

This is bounded by the right-hand side of (4.18). The stabilization term can be treated by the $L_{2}$-stability of $\kappa_{h}$ :

$$
\begin{aligned}
s_{h}\left(u-\mathcal{J}_{h} u, u-\mathcal{J}_{h} u\right) & \lesssim\left\|M_{p} \kappa_{h}\left(\nabla\left(p-\mathcal{J}_{h} p\right)\right)\right\|^{2}+\left\|M_{v} \kappa_{h}\left(\nabla\left(v-\mathcal{J}_{h} v\right)\right)\right\|^{2} \\
& \lesssim\left\|M_{p} \nabla\left(p-\mathcal{J}_{h} p\right)\right\|^{2}+\left\|M_{v} \nabla\left(v-\mathcal{J}_{h} v\right)\right\|^{2}
\end{aligned}
$$

The pressure part can be bounded by the interpolation properties of $\mathcal{J}_{h}$ by:

$$
\begin{aligned}
\left\|M_{p} \nabla\left(p-\mathcal{J}_{h} p\right)\right\|_{K}^{2} & =\alpha_{K, x}\left\|\partial_{x}\left(p-\mathcal{J}_{h} p\right)\right\|_{K}^{2}+\alpha_{K, y}\left\|\partial_{y}\left(p-\mathcal{J}_{h} p\right)\right\|_{K}^{2} \\
& \lesssim\left(\alpha_{K, x}+\alpha_{K, y} h_{K, y}^{-2} h_{K, x}^{2}\right) h_{K, x}^{2}\left\|\partial_{x} \nabla p\right\|_{K}^{2}+\left(\alpha_{K, x} h_{K, y}^{2} h_{K, x}^{-2}+\alpha_{K, y}\right) h_{K, y}^{2}\left\|\partial_{y} \nabla p\right\|_{K}^{2}
\end{aligned}
$$

Now we see that under the additional relation (4.17) between the parameters $\alpha_{x}$ and $\alpha_{y}$, that these terms are bounded by the right-hand side of (4.18). The velocity part of the stabilization behaves absolutely in the same way.

Lemma 4.5. On each cell $K \in \mathcal{T}_{h}$ and its associated patch $P=P(K) \in \mathcal{T}_{2 h}$ it holds

$$
\begin{array}{rlrl}
\left\|\kappa_{h} \partial_{x} u\right\|_{P} & \lesssim h_{K, x}\left\|\partial_{x} \nabla u\right\|_{P} & \forall u \in H^{2}(P), \\
\left\|\kappa_{h} \partial_{y} u\right\|_{P} & \lesssim h_{K, x}\left\|\partial_{x y} u\right\|_{P}+h_{K, y}\left\|\partial_{y y} u\right\|_{P} & \forall u \in H^{2}(P), \\
\left\|\kappa_{h} u\right\|_{P} & \lesssim\|u\|_{P} & & \forall u \in L_{2}(P) .
\end{array}
$$


Proof. Due to a scaling argument it holds for $w=\partial_{x} u$ :

$$
\left\|\kappa_{h} \partial_{x} u\right\|_{P}=\left\|w-\pi_{h} w\right\|_{P} \lesssim h_{P, x}\left\|\partial_{x} w\right\|_{P}+h_{P, y}\left\|\partial_{y} w\right\|_{P} \leq h_{K, x}\left\|\partial_{x} \nabla u\right\|_{P} .
$$

The other estimates are obtained similarly.

The next lemma shows that the stabilization term evaluated at the discrete interpolant $\mathcal{J}_{h} u$ can be bounded by the right-hand side of (4.18) as well:

Lemma 4.6. For the stabilization term defined in (3.4) and the relation (4.17) for the stabilization parameters it holds:

$$
\begin{aligned}
s_{h}\left(\mathcal{J}_{h} u ; \mathcal{J}_{h} u\right) \lesssim & \sum_{K \in \mathcal{T}_{h}}\left\{\alpha_{K, x} h_{K, x}^{2}\left\|\partial_{x} \nabla p\right\|_{K}^{2}+\alpha_{K, y} h_{K, y}^{2}\left\|\partial_{y} \nabla p\right\|_{K}^{2}\right. \\
& \left.+\left(\sigma+\left(\mu+\delta_{y}\right) h_{K, y}^{-2}\right)\left(h_{K, x}^{4}\left\|\partial_{x} \nabla v\right\|_{K}^{2}+h_{K, y}^{4}\left\|\partial_{y} \nabla v\right\|_{K}^{2}\right)\right\} .
\end{aligned}
$$

Proof. First, we note that due to the triangle inequality and Young's inequality one obtains:

$$
s_{h}\left(\mathcal{J}_{h} u ; \mathcal{J}_{h} u\right) \leq 2\left(s_{h}\left(\mathcal{J}_{h} u-u ; \mathcal{J}_{h} u-u\right)+s_{h}(u ; u)\right) .
$$

Due to $s_{h}\left(\mathcal{J}_{h} u-u ; \mathcal{J}_{h} u-u\right) \leq\left\|\mathcal{J}_{h} u-u\right\|_{\text {lps }}$ and Lemma 4.4 the first part of the right-hand side of (4.19) is bounded properly. The velocity part of the last term in (4.19) can be bounded on cell $K$ by use of Lemma 4.5 by the second derivatives on the associated patch $P=P(K) \in \mathcal{T}_{2 h}$ :

$$
\begin{aligned}
\left\|M_{v} \kappa_{h} \nabla v\right\|_{K}^{2} & \lesssim \delta_{K, x}\left\|\kappa_{h} \partial_{x} v\right\|_{K}^{2}+\delta_{K, y}\left\|\kappa_{h} \partial_{y} v\right\|_{K}^{2} \\
& \leq \delta_{K, x} h_{K, x}^{2}\left\|\partial_{x} \nabla v\right\|_{P}^{2}+\delta_{K, y} h_{K, x}^{2}\left\|\partial_{x y} v\right\|_{P}^{2}+\delta_{K, y} h_{K, y}^{2}\left\|\partial_{y y} v\right\|_{P}^{2} \\
& \lesssim \delta_{K, x} h_{K, x}^{2}\left\|\partial_{x} \nabla v\right\|_{P}^{2}+\delta_{K, y} h_{K, y}^{2}\left\|\partial_{y} \nabla v\right\|_{P}^{2} .
\end{aligned}
$$

Hence, with the relation $\delta_{K, x}=\delta_{K, y} h_{K, x}^{2} h_{K, y}^{-2}$ and the same procedure for the pressure stabilization we obtain an appropriate bound also on $s_{h}(u ; u)$.

\subsection{A priori error estimate on anisotropic Cartesian meshes}

The following auxiliary result will be necessary in the following.

Lemma 4.7. For the choice (3.6)-(3.10) for the stabilization parameters it holds:

$$
\begin{aligned}
\min \left(\delta_{K, y}^{-1}, \mu^{-1}\right) & =\alpha_{K, x} h_{K, x}^{-2} \\
\max \left(\delta_{K, y}, \mu\right) & =\alpha_{K, y}^{-1} h_{K, y}^{2}
\end{aligned}
$$

Proof. We start with the square of the right-hand side of (4.20). Due to the assumption $h_{K, y} \leq h_{K, x}$ we have $\mathrm{Pe}_{\min , K}=\|\beta\|_{\infty, K} h_{K, y} \mu^{-1}$ and therefore:

$$
\alpha_{K, x} h_{K, x}^{-2}=\mu^{-1} \min \left(1, \mathrm{Pe}_{\min , K}^{-1}\right)=\min \left(\mu^{-1}, h_{K, y}^{-1}\|\beta\|_{\infty, K}^{-1}\right) .
$$

Furthermore, it holds:

$$
\begin{aligned}
\delta_{K, y} & =h_{K, y}^{2}\|\beta\|_{\infty, K}^{2} \mu^{-1} \min \left(1, \mathrm{Pe}_{\min , K}^{-1}\right) \\
& =h_{K, y}\|\beta\|_{\infty, K} \mathrm{Pe}_{\min , K} \min \left(1, \mathrm{Pe}_{\min , K}^{-1}\right) \\
& =h_{K, y}\|\beta\|_{\infty, K} \min \left(\mathrm{Pe}_{\min , K}, 1\right)
\end{aligned}
$$


Using this in (4.22) gives

$$
\begin{aligned}
\alpha_{K, x} h_{K, x}^{-2} & =\min \left(\mu^{-1}, \delta_{K, y}^{-1} \min \left(\mathrm{Pe}_{\min , K}, 1\right)\right) \\
& =\min \left(\mu^{-1}, \delta_{K, y}^{-1}, \delta_{K, y}^{-1} \mathrm{Pe}_{\min , K}\right)
\end{aligned}
$$

In the case of $\mathrm{Pe}_{\min , K} \geq 1$ this implies (4.20) directly. For $\mathrm{Pe}_{\min , K}<1$ this is also true, because it follows with (4.23): $\delta_{K, y}^{-1} \mathrm{Pe}_{\min , K}=h_{K, y}^{-1}\|\beta\|_{\infty, K}^{-1}=\mathrm{Pe}_{\min , K}^{-1} \mu^{-1}>\mu^{-1}$. The identity (4.21) follows now immediately by help of (4.20):

$$
\alpha_{K, y}^{-1} h_{K, y}^{2}=\alpha_{K, x}^{-1} h_{K, x}^{2}=\left(\min \left(\delta_{K, y}^{-1}, \mu^{-1}\right)\right)^{-1}=\max \left(\delta_{K, y}, \mu\right) .
$$

Theorem 4.8. For the choice of the stabilization parameters as in (3.6)-(3.10) we obtain:

$$
\begin{aligned}
\left\|u-u_{h}\right\|_{\mathrm{lps}}^{2} \lesssim \sum_{K \in \mathcal{T}_{h}}\{ & h_{K, x}^{4} h_{K, y}^{-1}\left(C_{K, p}\left\|\partial_{x} \nabla p\right\|_{K}^{2}+C_{K, v}\left\|\partial_{x} \nabla v\right\|_{K}^{2}\right) \\
& \left.+h_{K, y}^{3}\left(C_{K, p}\left\|\partial_{y} \nabla p\right\|_{K}^{2}+C_{K, v}\left\|\partial_{y} \nabla v\right\|_{K}^{2}\right)\right\} .
\end{aligned}
$$

with

$$
\begin{aligned}
& C_{K, p}=\min \left(\mathrm{Pe}_{\min , \mathrm{K}}, 1\right)\|\beta\|_{K, \infty}^{-1}, \\
& C_{K, v}=h_{K, y} \sigma+\mu h_{K, y}^{-1}+\|\beta\|_{K, \infty} .
\end{aligned}
$$

Proof. We split the error $u-u_{h}$ into the interpolation part $\eta=u-\mathcal{J}_{h} u$ and the projection part $\xi=\mathcal{J}_{h} u-u_{h}$. The interpolation part is bounded by Lemma 4.4. It is easy to check that for the choice of parameters as in (3.6)-(3.10) we obtain $\alpha_{0}=C_{K, p} / h_{K, y}$ and $\left(\sigma+\left(\mu+\delta_{K, y}\right) h_{K, y}^{-2}\right) \leq C_{K, v} / h_{K, y}$. The interpolation error $\|\eta\|_{\text {lps }}$ is therefore bounded by the right-hand side of (4.24). The sophisticated part is the projection error $\|\xi\|_{\text {lps }}$. Due to the stability result and the perturbed Galerkin orthogonality (Thm. 4.1) it holds:

$$
\begin{aligned}
\|\xi\|_{\text {lps }}^{2} & =a(\xi, \xi)+s_{h}(\xi, \xi) \\
& =-a(\eta, \xi)+a\left(u-u_{h}, \xi\right)+s_{h}(\xi, \xi) \\
& =-a(\eta, \xi)+s_{h}\left(u_{h}, \xi\right)+s_{h}(\xi, \xi) \\
& =-a(\eta, \xi)+s_{h}\left(\mathcal{J}_{h} u, \xi\right) .
\end{aligned}
$$

For the stabilization part we obtain:

$$
\begin{aligned}
\left|s_{h}\left(\mathcal{J}_{h} u, \xi\right)\right| & \leq s_{h}\left(\mathcal{J}_{h} u, \mathcal{J}_{h} u\right)^{1 / 2} s_{h}(\xi, \xi)^{1 / 2} \\
& \leq s_{h}\left(\mathcal{J}_{h} u, \mathcal{J}_{h} u\right)^{1 / 2}\|\xi\|_{\mathrm{lps}} .
\end{aligned}
$$

Hence, with Lemma 4.6 this contribution can be bounded appropriately. For the term $a(\eta, \xi)$ we have

$$
|a(\eta, \xi)| \lesssim\|\eta\|_{\text {lps }}\|\xi\|_{\text {lps }}+\left|\left(\eta_{p}, \operatorname{div} \xi^{v}\right)\right|+\left|\left(\eta_{v}, \nabla \xi^{p}\right)\right|+\left|\left(\eta_{v}, \beta \cdot \nabla \xi^{v}\right)\right|
$$

Therefore, it is sufficient to bound the last three terms of (4.25):

- Due to the orthogonality property (3.2) of $\pi_{h}$ it holds for the first term on a patch $K \in \mathcal{T}_{2 h}$ :

$$
\begin{aligned}
\left|\left(\eta_{p}, \operatorname{div} \xi^{v}\right)_{K}\right| & =\left|\left(\eta_{p},\left(\operatorname{div} \xi^{v}\right)-\pi_{h}\left(\operatorname{div} \xi^{v}\right)\right)_{K}\right| \\
& =\left|\left(\eta_{p}, \kappa_{h}\left(\operatorname{div} \xi^{v}\right)\right)_{K}\right| \\
& \leq \delta_{K, y}^{-1 / 2}\left\|\eta_{p}\right\|_{K}\left\|M_{v} \kappa_{h}\left(\nabla \xi^{v}\right)\right\|_{K} .
\end{aligned}
$$


In addition it holds

$$
\left|\left(\eta_{p}, \operatorname{div} \xi^{v}\right)_{K}\right| \leq \mu^{-1 / 2}\left\|\eta_{p}\right\|_{K} \mu^{1 / 2}\left\|\nabla \xi_{v}\right\|_{K} .
$$

Hence, the term $\left|\left(\eta_{p}, \operatorname{div} \xi^{v}\right)_{K}\right|$ is bounded by

$$
\left|\left(\eta_{p}, \operatorname{div} \xi^{v}\right)_{K}\right| \leq \gamma_{K}\left\|\eta_{p}\right\|_{K}\left(\mu^{1 / 2}\left\|\nabla \xi_{v}\right\|_{K}+\left\|M_{v} \kappa_{h}\left(\nabla \xi^{v}\right)\right\|_{K}\right),
$$

with $\gamma_{K}:=\min \left\{\delta_{K, y}^{-1 / 2}, \mu^{-1 / 2}\right\}$. According to Lemma 4.7 it holds $\gamma_{K}=\alpha_{K, x}^{1 / 2} h_{K, x}^{-1}$, so that

$$
\begin{aligned}
\left|\left(\eta_{p}, \operatorname{div} \xi^{v}\right)\right| & \leq\left(\sum_{K \in \mathcal{T}_{2 h}} \alpha_{K, x} h_{K, x}^{-2}\left\|\eta_{p}\right\|_{K}^{2}\right)\|\xi\|_{\text {lps }} \\
& \left.\leq\left(\sum_{K \in \mathcal{T}_{2 h}} \alpha_{K, x} h_{K, x}^{2}\left\|\partial_{x} \nabla p\right\|^{2}+\alpha_{K, y} h_{K, y}^{2}\left\|\partial_{y} \nabla p\right\|\right)^{2}\right)^{1 / 2}\|\xi\|_{\text {lps }} .
\end{aligned}
$$

- For the second term of (4.25) we have:

$$
\begin{aligned}
\left|\left(\eta_{v}, \nabla \xi^{p}\right)_{K}\right| & =\left|\left(\eta_{v}, \kappa_{h}\left(\nabla \xi^{p}\right)\right)_{K}\right| \\
& \lesssim\left(\alpha_{K, x}^{-1 / 2}\left\|\eta_{v_{1}}\right\|_{K}+\alpha_{K, y}^{-1 / 2}\left\|\eta_{v_{2}}\right\|_{K}\right)\left\|M_{p} \kappa_{h}\left(\nabla \xi^{p}\right)\right\|_{K} \\
& \lesssim \alpha_{K, y}^{-1 / 2}\left(h_{K, x}^{2}\left\|\partial_{x} \nabla v\right\|_{K}+h_{K, y}^{2}\left\|\partial_{y} \nabla v\right\|_{K}\right)\left\|M_{p} \kappa_{h}\left(\nabla \xi^{p}\right)\right\|_{K}
\end{aligned}
$$

Assembling these terms together gives:

$$
\left|\left(\eta_{v}, \nabla \xi^{p}\right)\right| \leq\left(\sum_{K \in \mathcal{T}_{2 h}} \frac{h_{K, y}}{\alpha_{K, y}}\left(h_{K, x}^{4} h_{K, y}^{-1}\left\|\partial_{x} \nabla v\right\|_{K}^{2}+h_{K, y}^{3}\left\|\partial_{y} \nabla v\right\|_{K}^{2}\right)\right)^{1 / 2}\|\xi\|_{\mathrm{lps}} .
$$

By help of Lemma 4.7 we have $\alpha_{K, y}^{-1} h_{K, y}=\max \left(\mu h_{K, y}^{-1},\|\beta\|_{K, \infty}\right) \leq C_{K, v}$, so that the considered term is bounded by the right hand side of (4.24).

- Similar arguments as for the first term gives for the third term of (4.25):

$$
\left|\left(\eta_{v}, \beta \cdot \nabla \xi^{v}\right)_{K}\right| \lesssim \gamma_{K}\|\beta\|_{K, \infty}\left\|\eta_{v}\right\|_{K}\left\|M_{v} \kappa_{h}\left(\nabla \xi^{v}\right)\right\|_{K},
$$

with $\gamma_{K}$ defined as before. Summing over all cells $K$ as for the previous terms and due to $\gamma_{K}^{2}\|\beta\|_{K, \infty}^{2} h_{y}=$ $\alpha_{K, 0} h_{y}\|\beta\|_{K, \infty}^{2}=\min \left(1, \mathrm{Pe}_{\min , \mathrm{K}}\right)\|\beta\|_{K, \infty} \leq C_{K, v}$ this term is also bounded by the right hand side of (4.24).

Assembling terms of equal order together leads to the assertion.

Remark 4.9. In contrast to residual-based stabilization methods the parameters $\alpha$ and $\beta$ are independent of the absorption coefficient $\sigma$ which can also be interpreted as the inverse of a time step, i.e. $\sigma \sim 1 / \Delta t$. This is due to the fact that the stabilization does not act on the zero-order term $\sigma v$ in the Oseen system.

Remark 4.10. Although the analysis holds for $r \geq 1$, it is only optimal for the case $r=1$. For $r \geq 2$ and higher regularity of the solution, the previous proof has to be extended. This is of course possible but the anisotropic character would make the presentation much more opaque. 


\subsection{A priori error estimate on isotropic meshes}

In the case of an isotropic mesh, $h_{K}=h_{K, x} \sim h_{K, y}$, the stabilization terms become $\left.\alpha\right|_{K}=\alpha_{K, x} \sim \alpha_{K, y}$ and $\left.\delta\right|_{K}=\delta_{K, x} \sim \delta_{K, y} \sim\|\beta\|_{K, \infty}^{2} \alpha_{K}$. Theorem 4.8 becomes in this special case:

Theorem 4.11. The a priori estimate becomes in the isotropic case:

$$
\left\|u-u_{h}\right\|_{\text {lps }}^{2} \lesssim \sum_{K \in \mathcal{T}_{h}} h_{K}^{3}\left(C_{K, p}\left\|\nabla^{2} p\right\|_{K}^{2}+C_{K, v}\left\|\nabla^{2} v\right\|^{2}\right)
$$

with $C_{K, p}=\min \left(\mathrm{Pe}_{K}, 1\right)\|\beta\|_{K, \infty}^{-1}$ and $C_{K, v}=h_{K} \sigma+\mu h_{K}^{-1}+\|\beta\|_{K, \infty}$.

In particular, for Peclet number larger than one, i.e. the convection dominated case, the right hand side becomes (formally) independent of the viscosity:

$$
\begin{aligned}
& C_{p}=\|\beta\|_{K, \infty}^{-1} \\
& C_{v} \lesssim h_{K} \sigma+\|\beta\|_{K, \infty} .
\end{aligned}
$$

\subsection{A priori estimate for rotated meshes}

In Section 3.3 we discussed the anisotropic LPS variant on rectangular meshes not necessarily aligned with the coordinate axes. In the particular case of rotated meshes, the transformation is of the type $T_{K}=t_{k}+\Lambda_{K} Z_{K}$, with $Z_{K}=\left[\eta_{K, 1}, \eta_{K, 2}\right]$ and $\Lambda_{K}=\operatorname{diag}\left(\lambda_{K, 1}, \lambda_{K, 2}\right)$. We obtain a similar a priori estimate as before in Theorem 4.8 by replacing the partial derivatives $\partial_{x}$ and $\partial_{y}$ by the partial derivatives in direction $\eta_{1}$ and $\eta_{2}$ of the turned coordinate system.

$$
\begin{aligned}
\left\|u-u_{h}\right\|_{\mathrm{lps}}^{2} \lesssim & \sum_{K \in \mathcal{T}_{h}}\left(\alpha_{K, \eta_{1}} \lambda_{K, 1}^{2}\left\|\partial_{\eta_{1}} \nabla p\right\|_{K}^{2}+\alpha_{K, \eta_{2}} \lambda_{K, 2}^{2}\left\|\partial_{\eta_{2}} \nabla p\right\|_{K}^{2}\right. \\
& \left.+\left(\sigma+\left(\mu+\delta_{K, \eta_{2}}\right) \lambda_{K, 2}^{-2}\right) \cdot\left(\lambda_{K, 1}^{4}\left\|\partial_{\eta_{1}} \nabla v\right\|_{K}^{2}+\lambda_{K, 2}^{4}\left\|\partial_{\eta_{2}} \nabla v\right\|_{K}^{2}\right)\right) .
\end{aligned}
$$

\section{NumERICAL VALIDATION}

In order to validate the proposed discrete scheme for the computation of convection dominated flows on anisotropic meshes we consider the nonlinear Navier-Stokes equations:

$$
\begin{aligned}
(v \cdot \nabla v)-\mu \Delta v+\nabla p & =f \\
\operatorname{div} v & =0
\end{aligned}
$$

together with boundary conditions for $v$ and $p$ specified later for the particular problem. We need configurations with the following properties of the solution $u=\left\{v_{1}, v_{2}, p\right\}$ for the stationary Navier-Stokes equations:

- $u$ should be analytically given in order to evaluate discretization errors;

- $u$ should have a boundary layer in order to justify anisotropic meshes.

We design here two test problems which will be used for numerical validation of the anisotropic stabilization.

The first configuration consists of a flow in a tube with a boundary layer on a Cartesian mesh. It turns out that up to a certain degree of anisotropy the classical isotropic local projection stabilization is still convergent. Therefore, we will make a comparison with the anisotropic version. The second configuration consists of a flow in a disc with a very sharp boundary layer. Here, the orientation of the mesh and the anisotropy depends on the particular cell. It turns out, that the (multigrid) solver does not converge any more on highly anisotropic meshes with isotropic LPS. However, with anisotropic stabilization, we obtain fast convergence and the theoretical results of the preceding sections are reflected. 


\subsection{Tube flow with boundary layer}

In this numerical test case we consider a tube with length $L \geq 1$ and height $H=1$. The computational domain is therefore given by $\Omega=(0, L) \times(0, H)$. We have Dirichlet conditions for the velocities

$$
v=v_{0} \text { on } \Gamma_{\text {dir }}
$$

at the boundary part $\Gamma_{\text {dir }} \subset \partial \Omega$ consisting of the horizontal and the left vertical part of $\partial \Omega$. The remaining boundary (right vertical boundary part) $\Gamma_{\text {out }}:=\{L\} \times(0,1)$ is the natural "outflow" boundary

$$
\mu \frac{\partial v}{\partial n}-p \cdot n=0 \text { on } \Gamma_{\text {out }} .
$$

The boundary values $v_{0}$ and the forcing term $f$ of the momentum equation (5.1) are taken in such a way that we obtain the following analytical solution:

$$
\begin{aligned}
v_{1}(x, y) & =\frac{\mathrm{e}^{\gamma}-\mathrm{e}^{\gamma(1-y)}}{\mathrm{e}^{\gamma}-1}, \\
v_{2}(x, y) & =\epsilon(1-x / L)^{2}, \\
p(x, y) & =(L-x) x y,
\end{aligned}
$$

with $\epsilon=0.01$ and $\gamma=\mu^{-1 / 2}$. The velocity component in $x$-direction, $v_{1}$, exhibits a boundary layer at the lower boundary $y=0$ of thickness $\gamma^{-1}=\sqrt{\mu}$. The vertical velocity is chosen small but non-zero so that the velocity stabilization in vertical direction does not vanish for the exact solution and so that $v_{2}$ is not in the discrete space $Q_{1, h}$.

We compare the classical isotropic local projection stabilization with the anisotropic version derived in the previous sections for the (interesting) convection dominant case $\mu=10^{-5}$. In order to see the impact of anisotropy we take the length as $L \in\{2,10,50,100\}$. The case $L=1$ needs not be performed because both methods become identical on isotropic meshes. The number of grid points are equal for both coordinate directions and equidistant in $x$-direction as well as in $y$-direction. Hence the anisotropy is uniform in space and equal to $a=L / H=L$. Because the computational domain $\Omega$ as well as $h_{x}$ increase linearly with the amount of anisotropy, also the discretization errors are expected to scale at least linearly in dependence of $a$.

Because we use Cartesian grids for this model problem, the stabilization can be chosen as proposed in the theoretical part of this work:

$$
\begin{array}{lll}
\alpha_{x}:=\alpha_{0} h_{x}^{2} \mu^{-1} \min \left(1, \mathrm{Pe}_{y}^{-1}\right), & \delta_{x}:=\|\beta\|_{\infty}^{2} \alpha_{x} \\
\alpha_{y}:=\alpha_{0} h_{y}^{2} \mu^{-1} \min \left(1, \mathrm{Pe}_{y}^{-1}\right), & \delta_{y}:=\|\beta\|_{\infty}^{2} \alpha_{y}
\end{array}
$$

The parameter $\alpha_{0}=1$ leads just to a uniform scaling.

The results for the moderate aspect ratio $a=2$ are shown in the Table 1 . Since the scaling of the two velocity components $v_{1}$ and $v_{2}$ as well as their gradients are very different, the error in each velocity component is listed separately. The isotropic and anisotropic version of the local projection stabilization behave pretty much the same. Whereas, a small increase of the error is observed in the horizontal velocity component by a factor of nearly two for the anisotropic version, the error in the vertical velocity component is reduced by more than a factor of 2. However, we observe $\mathcal{O}\left(h^{2}\right)$ convergence for the $L_{2}$-error in pressure and velocities with the isotropic version as well with the anisotropic version. The error in the gradient of $p, v_{1}$ and $v_{2}$ is at least of order $\mathcal{O}(h)$.

At aspect ratio $a=10$, see Table 2, the isotropic version shows its first deficits. Although both methods show the theoretically expected convergence rates $\mathcal{O}(h)$ and $\mathcal{O}\left(h^{2}\right)$, depending on the specific quantity, the error with the anisotropic version is of about three orders of magnitude smaller for the vertical velocity component. In the horizontal velocity component, the discretization error with anisotropic stabilization is also substantially reduced. Surprisingly, the pressure is even slightly better with the isotropic version by a very small factor. 
TABlE 1. Convergence history for tube flow with iso- and anisotropic stabilization for aspect ratio $a=2$ and $\mu=10^{-5}$.

\begin{tabular}{|c|c|c|c|c|c|c|}
\hline$h_{y}$ & $\left\|p-p_{h}\right\|$ & $\left\|\nabla\left(p-p_{h}\right)\right\|$ & $\left\|v_{1}-v_{1, h}\right\|$ & $\left\|\nabla\left(v_{1}-v_{1, h}\right)\right\|$ & $\left\|v_{2}-v_{2, h}\right\|$ & $\left\|\nabla\left(v_{2}-v_{2, h}\right)\right\|$ \\
\hline \multicolumn{7}{|c|}{ isotropic LPS } \\
\hline $1 / 2$ & $2.79 \mathrm{e}-01$ & $1.15 \mathrm{e}+00$ & $3.31 \mathrm{e}-02$ & $2.10 \mathrm{e}-01$ & $6.45 \mathrm{e}-04$ & $2.04 \mathrm{e}-03$ \\
\hline $1 / 4$ & $6.12 \mathrm{e}-02$ & $4.78 \mathrm{e}-01$ & $3.69 \mathrm{e}-02$ & $2.81 \mathrm{e}-01$ & $1.85 \mathrm{e}-02$ & $1.48 \mathrm{e}-01$ \\
\hline $1 / 8$ & $1.51 \mathrm{e}-02$ & $2.35 \mathrm{e}-01$ & $6.88 \mathrm{e}-03$ & $1.06 \mathrm{e}-01$ & $3.94 \mathrm{e}-03$ & $6.13 \mathrm{e}-02$ \\
\hline $1 / 16$ & $3.79 \mathrm{e}-03$ & $1.18 \mathrm{e}-01$ & $1.27 \mathrm{e}-03$ & $4.14 \mathrm{e}^{-}-22$ & $6.97 \mathrm{e}-04$ & $2.20 \mathrm{e}-02$ \\
\hline $1 / 32$ & $9.50 \mathrm{e}-04$ & $5.89 \mathrm{e}-02$ & $2.17 \mathrm{e}-04$ & $1.60 \mathrm{e}-02$ & $1.20 \mathrm{e}-04$ & $7.66 \mathrm{e}-03$ \\
\hline $1 / 64$ & $2.38 \mathrm{e}-04$ & $2.95 \mathrm{e}-02$ & $4.21 \mathrm{e}-05$ & $6.94 \mathrm{e}-03$ & $2.06 \mathrm{e}-05$ & $2.61 \mathrm{e}-03$ \\
\hline $1 / 128$ & $5.94 \mathrm{e}-05$ & $1.47 \mathrm{e}-02$ & $9.58 \mathrm{e}-06$ & $3.38 \mathrm{e}-03$ & $3.45 \mathrm{e}-06$ & $8.52 \mathrm{e}-04$ \\
\hline $1 / 256$ & $1.49 \mathrm{e}-05$ & $7.37 \mathrm{e}-03$ & $2.24 \mathrm{e}-06$ & $1.67 \mathrm{e}-03$ & $5.25 \mathrm{e}-07$ & $2.42 \mathrm{e}-04$ \\
\hline \multicolumn{7}{|c|}{ anisotropic LPS } \\
\hline $1 / 2$ & $4.89 \mathrm{e}-01$ & $1.21 \mathrm{e}+00$ & $3.31 \mathrm{e}-02$ & $2.10 \mathrm{e}-01$ & $6.45 \mathrm{e}-04$ & $2.04 \mathrm{e}-03$ \\
\hline $1 / 4$ & $6.50 \mathrm{e}-02$ & $5.00 \mathrm{e}-01$ & $1.90 \mathrm{e}-02$ & $2.04 \mathrm{e}-01$ & $3.27 \mathrm{e}-03$ & $2.43 \mathrm{e}-02$ \\
\hline $1 / 8$ & $1.64 \mathrm{e}-02$ & $2.46 \mathrm{e}-01$ & $4.56 \mathrm{e}-03$ & $1.05 \mathrm{e}-01$ & $4.80 \mathrm{e}-04$ & $6.08 \mathrm{e}-03$ \\
\hline $1 / 16$ & $4.13 \mathrm{e}-03$ & $1.23 \mathrm{e}-01$ & $1.08 \mathrm{e}-03$ & $5.29 \mathrm{e}-02$ & $6.88 \mathrm{e}-05$ & $1.60 \mathrm{e}-03$ \\
\hline $1 / 32$ & $1.03 \mathrm{e}-03$ & $6.15 \mathrm{e}-02$ & $2.61 \mathrm{e}-04$ & $2.65 \mathrm{e}-02$ & $1.22 \mathrm{e}-05$ & $4.67 \mathrm{e}-04$ \\
\hline $1 / 64$ & $2.59 \mathrm{e}-04$ & $3.08 \mathrm{e}-02$ & $6.63 \mathrm{e}-05$ & $1.32 \mathrm{e}-02$ & $3.21 \mathrm{e}-06$ & $1.65 \mathrm{e}-04$ \\
\hline $1 / 128$ & $6.47 \mathrm{e}-05$ & $1.54 \mathrm{e}-02$ & $1.68 \mathrm{e}-05$ & $6.63 \mathrm{e}-03$ & $8.38 \mathrm{e}-07$ & $7.02 \mathrm{e}-05$ \\
\hline $1 / 256$ & $1.62 \mathrm{e}-05$ & $7.70 \mathrm{e}-03$ & $4.22 \mathrm{e}-06$ & $3.31 \mathrm{e}-03$ & $2.11 \mathrm{e}-07$ & $3.31 \mathrm{e}-05$ \\
\hline
\end{tabular}

TABLE 2. Convergence history for tube flow with iso- and anisotropic stabilization for aspect ratio $a=10$ and $\mu=10^{-5}$.

\begin{tabular}{r|cr|rr|rr|}
\hline$h_{y}$ & $\left\|p-p_{h}\right\|$ & $\left\|\nabla\left(p-p_{h}\right)\right\|$ & $\left\|v_{1}-v_{1, h}\right\|$ & $\left\|\nabla\left(v_{1}-v_{1, h}\right)\right\|$ & $\left\|v_{2}-v_{2, h}\right\|$ & $\left\|\nabla\left(v_{2}-v_{2, h}\right)\right\|$ \\
\hline \hline \multicolumn{7}{c}{ isotropic LPS } \\
\hline $1 / 2$ & $1.56 \mathrm{e}+01$ & $3.70 \mathrm{e}+01$ & $7.40 \mathrm{e}-02$ & $4.69 \mathrm{e}-01$ & $1.44 \mathrm{e}-03$ & $9.13 \mathrm{e}-04$ \\
$1 / 4$ & $3.60 \mathrm{e}+00$ & $8.81 \mathrm{e}+00$ & $8.01 \mathrm{e}-01$ & $5.04 \mathrm{e}+00$ & $1.14 \mathrm{e}+00$ & $5.32 \mathrm{e}+00$ \\
$1 / 8$ & $8.32 \mathrm{e}-01$ & $2.87 \mathrm{e}+00$ & $5.52 \mathrm{e}-01$ & $3.90 \mathrm{e}+00$ & $5.64 \mathrm{e}-01$ & $3.12 \mathrm{e}+00$ \\
$1 / 16$ & $1.97 \mathrm{e}-01$ & $1.22 \mathrm{e}+00$ & $2.34 \mathrm{e}-01$ & $2.60 \mathrm{e}+00$ & $1.59 \mathrm{e}-01$ & $1.58 \mathrm{e}+00$ \\
$1 / 32$ & $5.59 \mathrm{e}-02$ & $6.23 \mathrm{e}-01$ & $6.75 \mathrm{e}-02$ & $1.60 \mathrm{e}+00$ & $3.50 \mathrm{e}-02$ & $8.31 \mathrm{e}-01$ \\
$1 / 64$ & $1.45 \mathrm{e}-02$ & $3.23 \mathrm{e}-01$ & $1.29 \mathrm{e}-02$ & $8.12 \mathrm{e}-01$ & $6.53 \mathrm{e}-03$ & $3.39 \mathrm{e}-01$ \\
$1 / 128$ & $3.34 \mathrm{e}-03$ & $1.64 \mathrm{e}-01$ & $1.71 \mathrm{e}-03$ & $1.71 \mathrm{e}-01$ & $1.08 \mathrm{e}-03$ & $8.95 \mathrm{e}-02$ \\
$1 / 256$ & $8.29 \mathrm{e}-04$ & $8.22 \mathrm{e}-02$ & $1.83 \mathrm{e}-04$ & $2.62 \mathrm{e}-02$ & $1.65 \mathrm{e}-04$ & $2.04 \mathrm{e}-02$ \\
\hline \multicolumn{7}{|c|}{ anisotropic LPS } \\
\hline $1 / 2$ & $1.62 \mathrm{e}+01$ & $3.70 \mathrm{e}+01$ & $7.40 \mathrm{e}-02$ & $4.69 \mathrm{e}-01$ & $1.44 \mathrm{e}-03$ & $9.13 \mathrm{e}-04$ \\
$1 / 4$ & $3.49 \mathrm{e}+00$ & $8.36 \mathrm{e}+00$ & $4.77 \mathrm{e}-02$ & $4.82 \mathrm{e}-01$ & $8.64 \mathrm{e}-02$ & $3.58 \mathrm{e}-01$ \\
$1 / 8$ & $8.51 \mathrm{e}-01$ & $3.03 \mathrm{e}+00$ & $4.86 \mathrm{e}-02$ & $3.99 \mathrm{e}-01$ & $7.36 \mathrm{e}-03$ & $4.05 \mathrm{e}-02$ \\
$1 / 16$ & $2.13 \mathrm{e}-01$ & $1.37 \mathrm{e}+00$ & $3.82 \mathrm{e}-03$ & $1.25 \mathrm{e}-01$ & $5.22 \mathrm{e}-04$ & $4.29 \mathrm{e}-03$ \\
$1 / 32$ & $5.32 \mathrm{e}-02$ & $6.65 \mathrm{e}-01$ & $7.06 \mathrm{e}-04$ & $5.96 \mathrm{e}-02$ & $4.37 \mathrm{e}-05$ & $5.73 \mathrm{e}-04$ \\
$1 / 64$ & $1.33 \mathrm{e}-02$ & $3.30 \mathrm{e}-01$ & $1.73 \mathrm{e}-04$ & $2.97 \mathrm{e}-02$ & $6.49 \mathrm{e}-06$ & $1.19 \mathrm{e}-04$ \\
$1 / 128$ & $3.32 \mathrm{e}-03$ & $1.65 \mathrm{e}-01$ & $4.34 \mathrm{e}-05$ & $1.48 \mathrm{e}-02$ & $1.45 \mathrm{e}-06$ & $3.85 \mathrm{e}-05$ \\
$1 / 256$ & $8.31 \mathrm{e}-04$ & $8.24 \mathrm{e}-02$ & $1.09 \mathrm{e}-05$ & $7.41 \mathrm{e}-03$ & $3.54 \mathrm{e}-07$ & $1.60 \mathrm{e}-05$ \\
\hline
\end{tabular}


TABLE 3. Convergence history for tube flow with anisotropic stabilization for aspect ratio $a=50$ and $\mu=10^{-5}$. The isotropic version did not converge.

\begin{tabular}{r|cr|rr|rr|}
\hline$h_{y}$ & $\left\|p-p_{h}\right\|$ & $\left\|\nabla\left(p-p_{h}\right)\right\|$ & $\left\|v_{1}-v_{1, h}\right\|$ & $\left\|\nabla\left(v_{1}-v_{1, h}\right)\right\|$ & $\left\|v_{2}-v_{2, h}\right\|$ & $\left\|\nabla\left(v_{2}-v_{2, h}\right)\right\|$ \\
\hline \hline & anisotropic LPS \\
\hline $1 / 2$ & $1.52 \mathrm{e}+03$ & $1.98 \mathrm{e}+03$ & $1.65 \mathrm{e}-01$ & $1.05 \mathrm{e}+00$ & $3.23 \mathrm{e}-03$ & $4.08 \mathrm{e}-04$ \\
$1 / 4$ & $1.96 \mathrm{e}+02$ & $3.68 \mathrm{e}+02$ & $9.83 \mathrm{e}-02$ & $1.04 \mathrm{e}+00$ & $1.96 \mathrm{e}-01$ & $7.72 \mathrm{e}-01$ \\
$1 / 8$ & $4.76 \mathrm{e}+01$ & $8.93 \mathrm{e}+01$ & $4.78 \mathrm{e}-01$ & $3.74 \mathrm{e}+00$ & $1.66 \mathrm{e}-02$ & $8.24 \mathrm{e}-02$ \\
$1 / 16$ & $1.19 \mathrm{e}+01$ & $2.54 \mathrm{e}+01$ & $2.24 \mathrm{e}-02$ & $4.73 \mathrm{e}-01$ & $1.14 \mathrm{e}-03$ & $7.66 \mathrm{e}-03$ \\
$1 / 32$ & $2.97 \mathrm{e}+00$ & $8.99 \mathrm{e}+00$ & $2.33 \mathrm{e}-03$ & $1.40 \mathrm{e}-01$ & $8.95 \mathrm{e}-05$ & $8.48 \mathrm{e}-04$ \\
$1 / 64$ & $7.43 \mathrm{e}-01$ & $3.90 \mathrm{e}+00$ & $5.02 \mathrm{e}-04$ & $6.66 \mathrm{e}-02$ & $1.13 \mathrm{e}-05$ & $1.40 \mathrm{e}-04$ \\
$1 / 128$ & $1.86 \mathrm{e}-01$ & $1.87 \mathrm{e}+00$ & $1.25 \mathrm{e}-04$ & $3.32 \mathrm{e}-02$ & $2.32 \mathrm{e}-06$ & $3.49 \mathrm{e}-05$ \\
\hline
\end{tabular}

TABLE 4. Convergence history for tube flow with anisotropic stabilization for aspect ratio $a=100$ and $\mu=10^{-5}$. The isotropic version did not converge.

\begin{tabular}{r|cr|rr|rr|}
\hline$h_{y}$ & $\left\|p-p_{h}\right\|$ & $\left\|\nabla\left(p-p_{h}\right)\right\|$ & $\left\|v_{1}-v_{1, h}\right\|$ & $\left\|\nabla\left(v_{1}-v_{1, h}\right)\right\|$ & $\left\|v_{2}-v_{2, h}\right\|$ & $\left\|\nabla\left(v_{2}-v_{2, h}\right)\right\|$ \\
\hline \hline & anisotropic LPS \\
\hline $1 / 2$ & $1.50 \mathrm{e}+04$ & $1.12 \mathrm{e}+04$ & $2.34 \mathrm{e}-01$ & $1.48 \mathrm{e}+00$ & $4.56 \mathrm{e}-03$ & $2.89 \mathrm{e}-04$ \\
$1 / 4$ & $1.13 \mathrm{e}+03$ & $2.06 \mathrm{e}+03$ & $1.38 \mathrm{e}-01$ & $1.47 \mathrm{e}+00$ & $2.77 \mathrm{e}-01$ & $1.09 \mathrm{e}+00$ \\
$1 / 8$ & $2.71 \mathrm{e}+02$ & $4.84 \mathrm{e}+02$ & $1.23 \mathrm{e}+00$ & $1.02 \mathrm{e}+01$ & $2.36 \mathrm{e}-02$ & $1.17 \mathrm{e}-01$ \\
$1 / 16$ & $6.73 \mathrm{e}+01$ & $1.24 \mathrm{e}+02$ & $4.71 \mathrm{e}-02$ & $9.20 \mathrm{e}-01$ & $1.61 \mathrm{e}-03$ & $1.08 \mathrm{e}-02$ \\
$1 / 32$ & $1.68 \mathrm{e}+01$ & $3.59 \mathrm{e}+01$ & $4.06 \mathrm{e}-03$ & $2.04 \mathrm{e}-01$ & $1.25 \mathrm{e}-04$ & $1.19 \mathrm{e}-03$ \\
$1 / 64$ & $4.20 \mathrm{e}+00$ & $1.27 \mathrm{e}+01$ & $8.05 \mathrm{e}-04$ & $9.45 \mathrm{e}-02$ & $1.49 \mathrm{e}-05$ & $1.85 \mathrm{e}-04$ \\
$1 / 128$ & $1.05 \mathrm{e}+00$ & $5.52 \mathrm{e}+00$ & $2.00 \mathrm{e}-04$ & $4.69 \mathrm{e}-02$ & $2.91 \mathrm{e}-06$ & $4.32 \mathrm{e}-05$ \\
\hline
\end{tabular}

A possible explanation is that the pressure does not has a boundary layer. However, the difference is in the range of $1 \%$ and hence not relevant.

For aspect ratios $a=50$ and $a=100$ convergence is obtained only with anisotropic LPS, see Tables 3 and 4 . It was not possible to obtain results with the classical (isotropic) LPS or with PSPG/SUPG without increasing $\alpha_{0}$ and $\delta_{0}$. But with the anisotropic version of LPS, we still get second order behavior of the error in $L_{2}$ and better than first order in the gradients.

From previous work we know that the classical combination of pressure stabilized Petrov-Galerkin (PSPG) with streamline upwind Petrov-Galerkin (SUPG) for Navier-Stokes behaves pretty much the same like isotropic local projection stabilization, we conclude that this anisotropic stabilization performs much better.

\subsection{Circular flow with boundary layer}

In the second configuration non Cartesian meshes are subject of investigation. We propose to consider the unit circle $\Omega=\left\{(x, y) \in \mathbb{R}^{2}: x^{2}+y^{2}<1\right\}$ and a solution with quite simple structure in cylinder coordinates, $r=\sqrt{x^{2}+y^{2}}$ for the radius, and $\omega=\arccos (x / r)$ for the angle. The divergence-free constraint in cylinder coordinates become

$$
\operatorname{div} v=\frac{1}{r} \frac{\partial}{\partial r}\left(r v_{r}\right)+\frac{1}{r} \frac{\partial v_{\omega}}{\partial \omega}=0 .
$$

A velocity field with $v_{\omega}=$ const. and $v_{r}=0$ is therefore divergence free. A possible ansatz is:

$$
\begin{aligned}
& v_{1}(r, \omega)=-V(r) \sin (\omega), \\
& v_{2}(r, \omega)=V(r) \cos (\omega),
\end{aligned}
$$



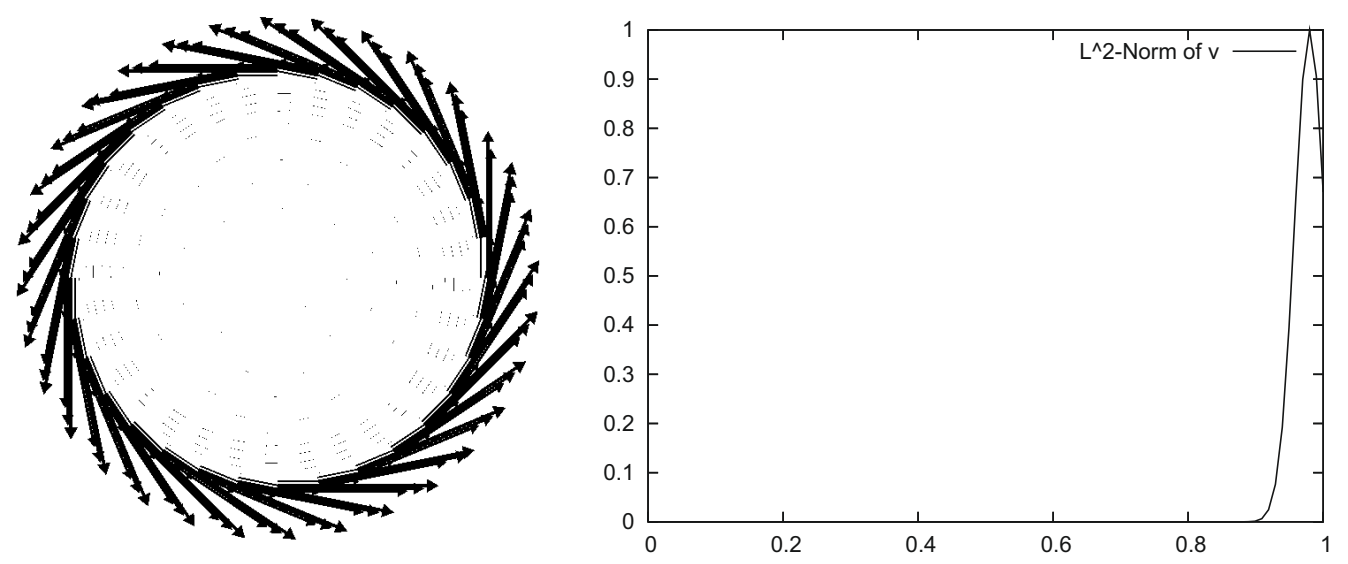

Figure 2. Left: Hedge hogs of the solution $v$. Right: $\|v\|$ in dependence of the radius $r$.

with $V(r)$ still to determined. In order to produce a boundary layer we take $V(r)=\mathrm{e}^{-\left(r-r_{0}\right)^{2} / \epsilon}$ with parameters $\epsilon=10^{-3}$ for tuning the size and $r_{0}=0.98$ for position the biggest variance in velocity very close to the boundary. Due to the Dirichlet conditions for the velocities the pressure has to be normalized. We take

$$
p(r, \omega)=r-\frac{2}{3},
$$

so that

$$
\int_{\Omega} p(x, y) \mathrm{d} x \mathrm{~d} y=\int_{0}^{1} \int_{0}^{2 \pi} r p(r, \omega) \mathrm{d} \omega \mathrm{d} r=0 .
$$

Hence, the pressure has zero mean, $\int_{\Omega} p \mathrm{~d} x=0$. The Dirichlet conditions for $v$ are given by

$$
v_{0}=V(1)\{-\sin (\omega), \cos (\omega)\} .
$$

The viscosity is taken as $\mu=10^{-2}$. Note, that the (exact) solution is independent of $\mu$, but one may also couple $\epsilon$ in dependence of $\mu$ when studies on varying viscosities are intended.

The velocity field in the disc are also illustrated in the left part of Figure 2, but the boundary layer can not be observed on that plot. Therefore, we show on the right part of Figure 2 the $L_{2}$-norm of the velocity, $\|v\|$, in dependence of the radius $r$ (this quantity is independent of $\omega$ ). Furthermore, the pressure field and the velocity field $v_{1}$ are shown in a 3-dimensional plot in Figure 3. Here, the vertical direction shows the corresponding value. Also here, the boundary layer in $v$ can be easily observed. An anisotropic mesh close to the boundary seems to be appropriate for resolving the sharp layer in $v$.

We perform two types of test:

(a) Simple bisection of all cells starting with different anisotropies at the outer circle. During the refinement process the degree of anisotropy is constant.

(b) We perform global refinement but we shift the mesh points during refinement towards the boundary layer. Hence, the degree of anisotropy increases under mesh refinement. In Figure 4, a zoom of such a mesh is shown.

The refinement strategy with a fixed anisotropy ratio (a) is chosen in order to validate the asymptotic convergence rate under mesh refinement with bisection. For (b) we expect a different convergence behavior for the errors in $p$ than in $v$. Since $v$ has only contributions close to the boundary we expect a better convergence in the velocities than in pressure. 

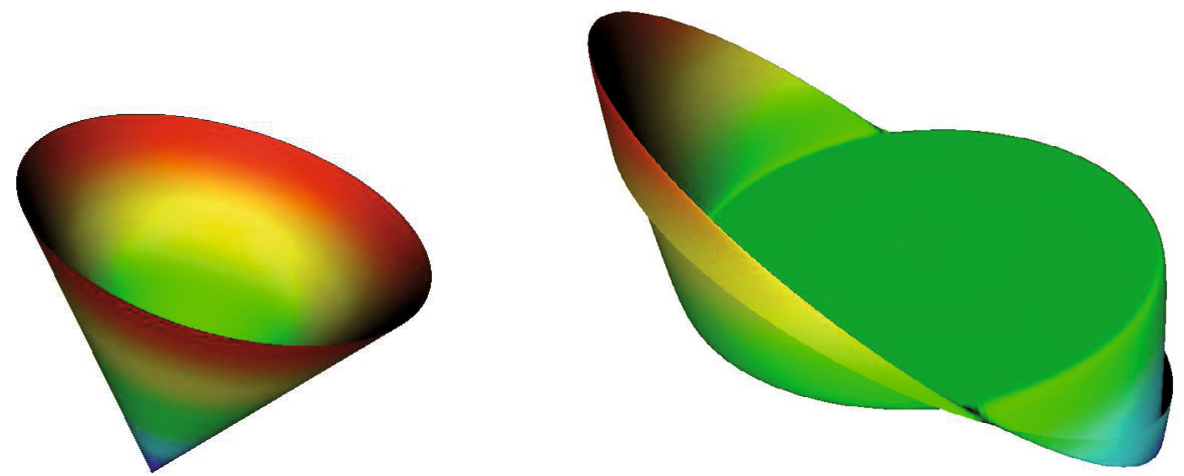

Figure 3. Pressure field and $x$-component of the velocity field, $v_{1}$.

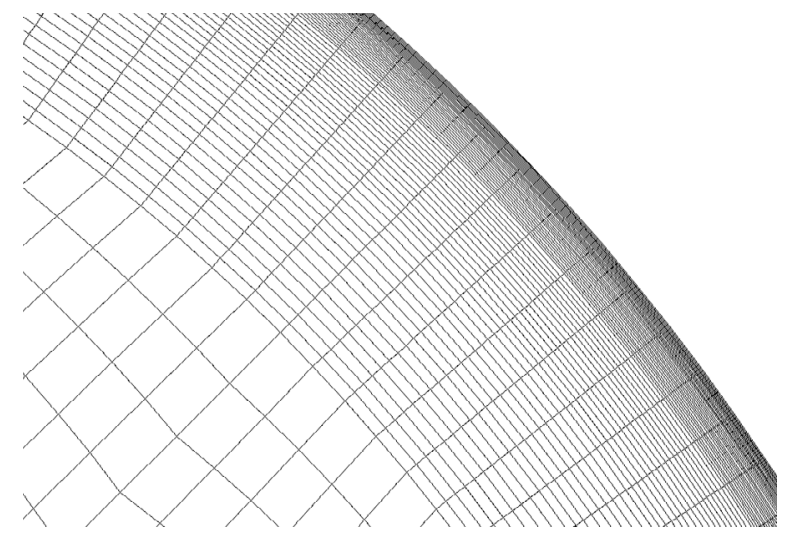

FiguRE 4. Zoom of a mesh used for solving the problem of circular flow.

In Table 5 the errors are presented for anisotropy ratio of 3, 5.25 and 16 for refinement strategy (a). Since the two components of the velocity are of the same character, we do not list their errors for each component, but the norms of the vectors $v-v_{h}$. All quantities show the expected convergence rates. Also here, the isotropic version of local projection does not work, so that we can not list the corresponding discretization errors.

The results with anisotropic LPS and refinement strategy (b) is listed in Table 6. The $L_{2}$-error of $p$ becomes almost divided by a factor of 4 from one mesh to the next, the error in the gradient, $\left\|\nabla\left(p-p_{h}\right)\right\|$ is almost halved. We do not approach the full factors 4 and 2, because of the already mentioned special designed refinement process (in order to increase the anisotropy gradually). The convergence of $\left\|v-v_{h}\right\|$ is a little bit better than $(\# \text { nodes })^{-1}$ and for $\left\|\nabla\left(v-v_{h}\right)\right\|$ better than (\# nodes $)^{-1 / 2}$. Hence, we see exactly the expected convergence behavior.

\section{Summary}

In this work, we extended the local projection stabilization for the Navier-Stokes discretization with equalorder bilinear finite elements to anisotropic quadrilateral meshes. Whereas on isotropic meshes we recover the classical local projection stabilization, we get a much more accurate and more robust method for highly anisotropic meshes, for instance when boundary layers should be resolved. We gave an a priori estimate for the Oseen linearization of the Navier-Stokes system leading to a qualitatively better convergence behavior than other isotropic stabilization methods. The numerical results reflect the theoretical expectations. 
TABLE 5. Convergence history for flow in a disc with anisotropic stabilization, Test (a).

\begin{tabular}{|c|c|c|c|c|}
\hline \# nodes & $\left\|p-p_{h}\right\|$ & $\nabla\left(p-p_{h}\right)$ & $\left\|v-v_{h}\right\|$ & $\left\|\nabla\left(v-v_{h}\right)\right\|$ \\
\hline & \multicolumn{3}{|c|}{ aspect ratio $=3$} & \\
\hline 25 & $3.56 \mathrm{e}-01$ & $2.97 \mathrm{e}+00$ & $6.30 \mathrm{e}-01$ & $8.37 \mathrm{e}+00$ \\
\hline 89 & $3.60 \mathrm{e}-01$ & $2.26 \mathrm{e}+00$ & $1.24 \mathrm{e}+00$ & $8.93 \mathrm{e}+00$ \\
\hline 337 & $8.99 \mathrm{e}-02$ & $7.52 \mathrm{e}-01$ & $6.54 \mathrm{e}-01$ & $1.20 \mathrm{e}+01$ \\
\hline 1313 & $6.20 \mathrm{e}-03$ & $1.98 \mathrm{e}-01$ & $2.08 \mathrm{e}-01$ & $1.13 \mathrm{e}+01$ \\
\hline 5185 & $1.78 \mathrm{e}-03$ & $1.25 \mathrm{e}-01$ & $6.91 \mathrm{e}-02$ & $6.97 \mathrm{e}+00$ \\
\hline 20609 & $5.26 \mathrm{e}-04$ & $3.83 \mathrm{e}-02$ & $1.73 \mathrm{e}-02$ & $3.47 \mathrm{e}+00$ \\
\hline \multirow[t]{2}{*}{82177} & $1.40 \mathrm{e}-04$ & $1.56 \mathrm{e}-02$ & $4.37 \mathrm{e}-03$ & $1.74 \mathrm{e}+00$ \\
\hline & \multicolumn{3}{|c|}{ aspect ratio $=5.25$} & \\
\hline 25 & $2.41 \mathrm{e}-01$ & $2.14 \mathrm{e}+00$ & $3.99 \mathrm{e}-01$ & $9.06 \mathrm{e}+00$ \\
\hline 89 & $3.44 \mathrm{e}-01$ & $5.77 \mathrm{e}+00$ & $3.08 \mathrm{e}-01$ & $1.02 \mathrm{e}+01$ \\
\hline 337 & $2.95 \mathrm{e}-02$ & $7.65 \mathrm{e}-01$ & $2.26 \mathrm{e}-01$ & $1.03 \mathrm{e}+01$ \\
\hline 1313 & $3.40 \mathrm{e}-03$ & $1.97 \mathrm{e}-01$ & $1.16 \mathrm{e}-01$ & $9.21 \mathrm{e}+00$ \\
\hline 5185 & $9.38 \mathrm{e}-04$ & $7.55 \mathrm{e}-02$ & $2.60 \mathrm{e}-02$ & $4.13 \mathrm{e}+00$ \\
\hline 20609 & $2.56 \mathrm{e}-04$ & $3.52 \mathrm{e}-02$ & $6.64 \mathrm{e}-03$ & $2.10 \mathrm{e}+00$ \\
\hline \multirow[t]{2}{*}{82177} & $6.78 \mathrm{e}-05$ & $1.84 \mathrm{e}-02$ & $1.65 \mathrm{e}-03$ & $1.04 \mathrm{e}+00$ \\
\hline & \multicolumn{3}{|c|}{ aspect ratio $=16$} & \\
\hline 25 & $4.31 \mathrm{e}-01$ & $6.54 \mathrm{e}+00$ & $3.97 \mathrm{e}-01$ & $6.65 \mathrm{e}+00$ \\
\hline 89 & $1.06 \mathrm{e}+00$ & $3.23 \mathrm{e}+01$ & $3.58 \mathrm{e}-01$ & $7.61 \mathrm{e}+00$ \\
\hline 337 & $5.31 \mathrm{e}-02$ & $2.92 \mathrm{e}+00$ & $1.08 \mathrm{e}-01$ & $5.60 \mathrm{e}+00$ \\
\hline 1313 & $2.57 \mathrm{e}-03$ & $1.44 \mathrm{e}-01$ & $2.45 \mathrm{e}-02$ & $2.83 \mathrm{e}+00$ \\
\hline 5185 & $7.35 \mathrm{e}-04$ & $7.77 \mathrm{e}-02$ & $6.49 \mathrm{e}-03$ & $1.41 \mathrm{e}+00$ \\
\hline 20609 & $2.07 \mathrm{e}-04$ & $4.23 \mathrm{e}-02$ & $1.83 \mathrm{e}-03$ & $6.97 \mathrm{e}-01$ \\
\hline 82177 & $5.92 \mathrm{e}-05$ & $2.30 \mathrm{e}-02$ & $4.76 \mathrm{e}-04$ & $3.38 \mathrm{e}-01$ \\
\hline
\end{tabular}

TABLE 6. Convergence history for flow in a disc with anisotropic stabilization, Test (b).

\begin{tabular}{|rc|rr|rr|}
\hline \# nodes & max. aspect ratio & $\left\|p-p_{h}\right\|$ & $\left\|\nabla\left(p-p_{h}\right)\right\|$ & $\left\|v-v_{h}\right\|$ & $\left\|\nabla\left(v-v_{h}\right)\right\|$ \\
\hline 25 & 3.6 & 0.298348 & 2.94098 & 0.34221 & 8.08301 \\
89 & 6.5 & 0.0464173 & 0.532067 & 0.473274 & 8.27074 \\
337 & 9.4 & 0.00721525 & 0.309655 & 0.116721 & 6.26525 \\
1313 & 12.0 & 0.0020788 & 0.137325 & 0.0332289 & 3.3837 \\
5185 & 20.4 & 0.000605623 & 0.0681489 & $7.73223 \mathrm{e}-03$ & 1.44632 \\
20609 & 40.7 & 0.000174225 & 0.0369805 & $1.92249 \mathrm{e}-03$ & 0.724083 \\
82177 & 81.4 & $5.43015 \mathrm{e}-05$ & 0.0209124 & $4.54694 \mathrm{e}-04$ & 0.340097 \\
\hline
\end{tabular}

\section{REFERENCES}

[1] T. Apel, Anisotropic finite elements: Local estimates and applications, Advances in Numerical Mathematics. Teubner, Stuttgart (1999).

[2] R. Becker, An adaptive finite element method for the incompressible Navier-Stokes equation on time-dependent domains. Ph.D. Dissertation, SFB-359 Preprint 95-44, Universität Heidelberg, Germany (1995).

[3] R. Becker and M. Braack, A finite element pressure gradient stabilization for the Stokes equations based on local projections. Calcolo 38 (2001) 173-199.

[4] R. Becker and M. Braack, A two-level stabilization scheme for the Navier-Stokes equations, in Numerical Mathematics and Advanced Applications, ENUMATH 2003, E.A.M. Feistauer Ed., Springer (2004) 123-130. 
[5] R. Becker, M. Braack and B. Vexler, Numerical parameter estimaton for chemical models in multidimensional reactive flows. Combust. Theory Model. 8 (2004) 661-682.

[6] R. Becker, M. Braack and B. Vexler, Parameter identification for chemical models in combustion problems. Appl. Numer. Math. 54 (2005) 519-536.

[7] M. Braack, Anisotropic $H^{1}$-stable projections on quadrilateral meshes, in Numerical Mathematics and Advanced Applications, Enumath Proc. 2005, B. de Castro Ed., Springer (2006) 495-503.

[8] M. Braack and E. Burman, Local projection stabilization for the Oseen problem and its interpretation as a variational multiscale method. SIAM J. Numer. Anal. 43 (2006) 2544-2566.

[9] M. Braack and T. Richter, Local projection stabilization for the Stokes system on anisotropic quadrilateral meshes, in Numerical Mathematics and Advanced Applications, Enumath Proc. 2005, B. de Castro Ed., Springer (2006) $770-778$.

[10] M. Braack and T. Richter, Solutions of 3D Navier-Stokes benchmark problems with adaptive finite elements. Comput. Fluids 35 (2006) 372-392.

[11] M. Braack and T. Richter, Stabilized finite elements for 3D reactive flow. Int. J. Numer. Methods Fluids 51 (2006) $981-999$.

[12] M. Braack, E. Burman, V. John and G. Lube, Stabilized finite element methods for the generalized Oseen problem. Comput. Methods Appl. Mech. Engrg. 196 (2007) 853-866.

[13] A. Brooks and T. Hughes, Streamline upwind Petrov-Galerkin formulation for convection dominated flows with particular emphasis on the incompressible Navier-Stokes equations. Comput. Methods Appl. Mech. Engrg. 32 (1982) $199-259$.

[14] E. Burman, M. Fernandez and P. Hansbo, Edge stabilization for the incompressible Navier-Stokes equations: a continuous interior penalty finite element method. SIAM J. Numer. Anal. 44 (2006) 1248-1274.

[15] P. Ciarlet, Finite Element Methods for Elliptic Problems. North-Holland, Amsterdam (1978).

[16] R. Codina, Stabilization of incompressibility and convection through orthogonal subscales in finite element methods. Comput. Methods Appl. Mech. Engrg. 190 (2000) 1579-1599.

[17] R. Codina and O. Soto, Approximation of the incompressible Navier-Stokes equations using orthogonal subscale stabilization and pressure segregation on anisotropic finite element meshes. Comput. Methods Appl. Mech. Engrg. 193 (2004) $1403-1419$.

[18] L. Formaggia and S. Perotto, Anisotropic error estimates for elliptic problems. Numer. Math. 94 (2003) 67-92.

[19] L. Formaggia, S. Micheletti and S. Perotto, Anisotropic mesh adaptation in computational fluid dynamics: Application to the advection-diffusion-reaction and the Stokes problems. Appl. Numer. Math. 51 (2004) 511-533.

[20] L. Franca and S. Frey, Stabilized finite element methods: II. The incompressible Navier-Stokes equations. Comput. Methods Appl. Mech. Engrg. 99 (1992) 209-233.

[21] J.-L. Guermond, Stabilization of Galerkin approximations of transport equations by subgrid modeling. ESAIM: M2AN 33 (1999) 1293-1316.

[22] P. Hansbo and A. Szepessy, A velocity-pressure streamline diffusion finite element method for the incompressible Navier-Stokes equations. Comput. Methods Appl. Mech. Engrg. 84 (1990) 175-192.

[23] T. Hughes, L. Franca and M. Balestra, A new finite element formulation for computational fluid dynamics: V. Circumvent the Babuska-Brezzi condition: A stable Petrov-Galerkin formulation for the Stokes problem accommodating equal order interpolation. Comput. Methods Appl. Mech. Engrg. 59 (1986) 89-99.

[24] V. John and S. Kaya, A finite element variational multiscale method for the Navier-Stokes equations. SIAM J. Sci. Comp. 26 (2005) 1485-1503.

[25] V. John, S. Kaya and W. Layton, A two-level variational multiscale method for convection-dominated convection-diffusion equations. Comput. Methods Appl. Mech. Engrg. 26 (2005) 4594-4603.

[26] K. Kunisch and B. Vexler, Optimal vortex reduction for instationary flows based on translation invariant cost functionals. SIAM J. Contr. Opt. 46 (2007) 1368-1397.

[27] T. Linss, Anisotropic meshes and streamline-diffusion stabilization for convection-diffusion problems. Comm. Numer. Methods Engrg. 21 (2005) 515-525.

[28] G. Lube and T. Apel, Anisotropic mesh refinement in stabilized Galerkin methods. Numer. Math. 74 (1996) $261-282$.

[29] G. Lube and G. Rapin, Residual-based stabilized higher-order FEM for a generalized Oseen problem. Math. Models Methods Appl. Sci. 16 (2006) 949-966.

[30] G. Lube, T. Knopp and R. Gritzki, Stabilized FEM with anisotropic mesh refinement for the Oseen problem, in Proceedings ENUMATH 2005, Springer (2006) 799-806.

[31] G. Matthies, P. Skrzypacz and L. Tobiska, A unified convergence analysis for local projection stabilisations applied ro the Oseen problem. ESAIM: M2AN 41 (2007) 713-742.

[32] S. Micheletti, S. Perotto and M. Picasso, Stabilized finite elements on anisotropic meshes: A priori estimate for the advectiondiffusion and the Stokes problem. SIAM J. Numer. Anal. 41 (2003) 1131-1162.

[33] H. Paillere, P. Le Quéré, C. Weisman, J. Vierendeels, E. Dick, M. Braack, F. Dabbene, A. Beccantini, E. Studer, T. Kloczko, C. Corre, V. Heuveline, M. Darbandi and S. Hosseinizadeh, Modelling of natural convection flows with large temperature differences: a benchmark problem for low Mach number solvers. Part 2. Contributions to the June 2004 conference. ESAIM: M2AN 39 (2005) 617-621.

[34] L. Tobiska and G. Lube, A modified streamline diffusion method for solving the stationary Navier-Stokes equations. Numer. Math. 59 (1991) 13-29. 